SubmitTed to AAS Journals, 1 OCTOBER 2017

Preprint typeset using LTEX style emulateapj v. 12/16/11

\title{
THE GRISM LENS-AMPLIFIED SURVEY FROM SPACE (GLASS). XII. SPATIALLY RESOLVED GALAXY STAR FORMATION HISTORIES AND TRUE EVOLUTIONARY PATHS AT $z>1^{*}$
}

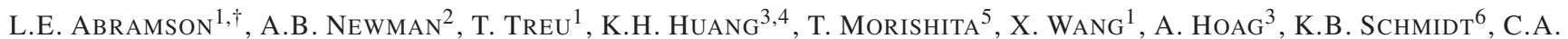

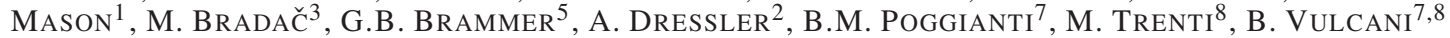 \\ Submitted to AAS Journals, 1 October 2017
}

\begin{abstract}
Modern data empower observers to describe galaxies as the spatially and biographically complex objects they are. We illustrate this through case studies of four, $z \sim 1.3$ systems based on deep, spatially resolved, 17-band + G102 + G141 Hubble Space Telescope grism spectrophotometry. Using full spectrum rest-UV/optical continuum fitting, we characterize these galaxies' observed $\sim \mathrm{kpc}$-scale structures and star formation rates (SFRs) and reconstruct their history over the age of the universe. The sample's diversity - passive to vigorously starforming; stellar masses $\log M_{*} / \mathrm{M}_{\odot}=10.5$ to 11.2 - enables us to draw spatio-temporal inferences relevant to key areas of parameter space (Milky Way- to super-Andromeda-mass progenitors). Specifically, we find signs that bulge mass-fractions $(B / T)$ and SF history shapes/spatial uniformity are linked, such that higher $B / T$ s correlate with "inside-out growth" and central specific SFRs that peaked above the global average for all starforming galaxies at that epoch. Conversely, the system with the lowest $B / T$ had a flat, spatially uniform SFH with normal peak activity. Both findings are consistent with models positing a feedback-driven connection between bulge formation and the switch from rising to falling SFRs ("quenching"). While sample size forces this conclusion to remain tentative, this work provides a proof-of-concept for future efforts to refine or refute it: JWST, WFIRST, and the $30 \mathrm{~m}$ class telescopes will routinely produce data amenable to this and more sophisticated analyses. These samples - spanning representative mass, redshift, SFR, and environmental regimes - will be ripe for converting into thousands of sub-galactic-scale empirical windows on what individual systems actually looked like in the past, ushering in a new dialog between observation and theory.
\end{abstract}

Subject headings: galaxies: evolution — galaxies: structure — galaxies: spectrophotometry

\section{INTRODUCTION}

A core goal of studying galaxy evolution is understanding why a galaxy has a given stellar mass $\left(M_{*}\right)$, star formation rate (SFR), size $\left(r_{e}\right)$, color, structure, and chemical composition at a given time. Large amounts of data describing those properties in different galaxies at different times circumscribe this endeavor: Deep and wide ground- and space-based imaging yields censuses to $z \gtrsim 2-$ over $75 \%$ of cosmic historycomplete to below the mass of the modern Milky Way (Grogin et al. 2011; Spitler et al. 2012; Illingworth et al. 2016). Ground- and space-based spectroscopy adds details on stellar populations, and (gaseous) metallicities, kinematics, and hydrodynamics to those images (York et al. 2000; Calvi et al. 2011; Oemler et al. 2013b; Kelson et al. 2014; Steidel et al. 2014; Shapley et al. 2015; Kriek et al. 2015; Brammer et al. 2012; Momcheva et al. 2016). Combined, the above form a rich database describing many aspects of the cosmic galaxy ensemble at different snapshots of its existence ready to be

1 UCLA, 430 Portola Plaza, Los Angeles, CA 90095, USA

2 The Carnegie Observatories, 813 Santa Barbara Street, Pasadena, CA 91101, USA

3 UC Davis, 1 Shields Avenue, Davis, CA 95616, USA

4 Translational \& Molecular Imaging Institute, Icahn School of Medicine at Mt. Sinai, 1470 Madison Avenue, New York, NY 10029, USA

5 Space Telescope Science Institute, 3700 San Martin Drive, Baltimore, MD 21218, USA

6 Leibniz-Institut für Astrophysik Potsdam, An der Sternwarte 16, 14482 Potsdam, Germany

7 INAF-Osservatorio Astronomico di Padova, Vicolo Osservatorio 5, I35122 Padova, Italy

8 School of Physics, University of Melbourne, VIC 3010, Australia

* This work incorporates data obtained using the Keck 10 m telescopes at Maunakea, Hawai'i.

$\dagger$ labramson@astro.ucla.edu compared to the ever-advancing numerical and semi-analytic models that seek to explain them (e.g., Benson 2012; Hopkins et al. 2014; Vogelsberger et al. 2014; McAlpine et al. 2016).

Indeed, at the population level, the mean and dispersion of many of the above spatially unresolved properties are probably well enough known to require little further empirical investigation: With samples of thousands of $\log M_{*} \gtrsim 10$, $z \lesssim 2$ systems, statistical uncertainties may no longer impede our understanding as much as systematic interpretive issues. $^{9}$ That is, knowing how the galaxy population evolves may never lead to a unique idea of how a galaxy developsthe objective stated above.

This problem arises from the inescapable fact that data are cross-sectional (different systems at different times) while theories are longitudinal (the same systems at different times). This allows different physical models to describe the same (uncontested) observations. Projections of the size-SFRmass plane provide rich grounds for such debate (Peng et al. 2010; Pacifici et al. 2013, 2016; Gladders et al. 2013; Kelson 2014; Zolotov et al. 2015; Barro et al. 2016; Abramson et al. 2016; Oemler et al. 2016; Lilly \& Carollo 2016; Morishita et al. 2017; Abramson \& Morishita 2016): Whether, for example, red galaxies' small size compared to blue ones reflects a rapid quenching transformation or merely the universe's density evolution has strong implications for the interplay between galactic structure and star formation.

Progress requires lifting these descriptive degeneracies using different kinds of information, not just more of it (Abramson et al. 2016; Kelson et al. 2016). Principally, higher resolution observations and inferences may be key.

\footnotetext{
${ }^{9}$ This is not true with respect to environment, the circumgalactic medium, neutral gas content, or stellar chemical abundances $(Z)$.
} 
Observationally, despite the breadth and depth of current surveys, galaxies are still typically analyzed as monolithic entities with a single stellar mass, SFR, or metallicity. As such, they are condensed to points in parameter space through (or near) which more than one physical model can pass.

The advent of large samples of spatially resolved integral field unit (IFU) spectroscopy is changing this norm. These data transform galaxies into many stellar populations corresponding to many mass surface densities/metallicities, challenging models to reproduce distributions for individual objects, not just integrated quantities (Blanc et al. 2009; Förster Schreiber et al. 2009; Cappellari et al. 2011; Sánchez et al. 2012; Allen et al. 2015; Bundy et al. 2015; Magdis et al. 2016; Goddard et al. 2017; Poggianti et al. 2017; Mason et al. 2017).

Inferentially, empirical reconstructions of galaxy star formation histories (SFHs) are adding new temporal resolution, shedding light on a system's full growth curve, not just its integral $\left(M_{*}\right)$ or current derivative $\left(\operatorname{SFR}\left(t_{\mathrm{obs}}\right)\right)$. This endeavor stretches back at least to Tinsley (1968) and has been used to great effect to study the light-/mass-weighted ages of red galaxies (constraining quenching timescales and late-time star formation; e.g., Dressler \& Gunn 1983; Couch \& Sharples 1987; Poggianti et al. 1999, 2013; Kelson et al. 2001; Kauffmann et al. 2003; Dressler et al. 2004; Treu et al. 2005; Fritz et al. 2007, 2014; Kriek et al. 2008; Domínguez Sánchez et al. 2016). However, it also provides insight into more abstract questions, such as the extended role of environment (Thomas et al. 2005; Trager et al. 2000; Kelson et al. 2006; Guglielmo et al. 2015; McDermid et al. 2015), whether galaxy histories are self-similar (Pacifici et al. 2016), how their parameterization affects population-level physical inferences (Tinsley 1968; Gallagher et al. 1984; Oemler et al. 2013a; Iyer \& Gawiser 2017; Ciesla et al. 2017), and whether established ways of classifying galaxies or matching them to dark matter halos require rethinking (Weisz et al. 2014; Dressler et al. 2016; Dressler et al., in preparation).

Expanding the space in which we think about galaxies and "longitudinalizing" their cross-sectional snapshots have great promise individually, but combining them is optimal: While higher spatial resolution data allow for more realistic descriptions, reconstructing SFHs for parts of galaxies would enable full spatio-spectral projections of what individual systems actually looked like in the past (as opposed to, e.g., abundance matching-based inferences; van Dokkum et al. 2013; Morishita et al. 2015). Unfortunately, due to resolution and signal-to-noise ratio $(S / N)$ requirements, suitable IFU surveys reaching stellar continua are limited to the local universe (Ma et al. 2014; McDermid et al. 2015; Goddard et al. 2017), far from the peak of stellar mass production/galaxy growth $(0.7 \lesssim z \lesssim 2$; Madau \& Dickinson 2014). Yet, by brightening and extending sources by $\sim 2-10 \times$, gravitational lensing can help bypass this constraint.

Combined with the native sensitivity and spatial resolution of the Hubble Space Telescope (HST), lensing-especially by foreground galaxy clusters-yields unprecedented views into galaxies at the peak of cosmic activity (e.g., Wuyts et al. 2012a,b), resolving their star formation and gas/stellar properties in such detail as to meaningfully challenge theory (Wuyts et al. 2014; Jones et al. 2015; Newman et al. 2015; Wang et al. 2017; Mason et al. 2017; Johnson et al. 2017a,b; Rigby et al. 2017; Toft et al. 2017). The Grism Lens-Amplified Survey from Space (GLASS; Schmidt et al. 2014; Treu et al. 2015) builds on this foundation, turning HST's slitless Wide Field Camera 3 (WFC3) IR grism spectrographs on the powerful lenses from the Cluster Lensing And Supernova survey with Hubble (CLASH; Postman et al. 2012) and Hubble Frontier Field (HFF; Lotz et al. 2017) campaigns.

Exploiting space's low IR backgrounds, lack of seeing limitations, and lensing's additional resolution and $S / N$ boosts, GLASS provides rest-optical continuum spectra for SFRunbiased, $z \gtrsim 1$ galaxy samples resolved out to $\sim 2$ half-light radii. These IFU-like data (Vulcani et al. 2015b, 2016, 2017; Wang et al. 2017) enable individual systems' size, mass, and SFR evolution to be inferred over Gyr timescales, providing new spatio-temporal empirical windows on how galaxies wove their way through the cosmic narrative.

Here, we use these data to reconstruct the spatially resolved histories of four $z>1, \log M_{*} \gtrsim 10.5$ galaxies spanning a diversity of star formation states from fully passive to strongly starforming. We then use these inferences to draw links between the systems' observed stellar structure and the shapes of their SF histories. While sample size limits the generality of our conclusions, our analysis represents a first step towards building a new common ground for observational and theoretical studies, providing a proof of concept for methods that could soon readily be applied to thousands of representative systems from JWST, WFIRST, and $30 \mathrm{~m}$ class facilities.

We proceed as follows: Section 2 describes the HST spectrophotometry and lensing magnification estimates (2.5); Section 3 the SFH fitting process; Section 4 the integrated (4.1), spatially resolved (4.2), and spatio-temporally resolved (4.3) inferences derived therefrom; and Section 5 their astrophysical (5.1), and methodological/theoretical (5.2) implications. Appendices A-E provide further details. Readers comfortable with modern spectral energy distribution (SED) fitting techniques can skip to Section 4.

We take $\left(H_{0}, \Omega_{m}, \Omega_{\Lambda}\right)=\left(73 \mathrm{kms}^{-1} \mathrm{Mpc}^{-1}, 0.27,0.73\right)$ and quote $\mathrm{AB}$ magnitudes.

\section{DATA}

We combine public GLASS spectroscopy ${ }^{10}$ with CLASH and HFF imaging ${ }^{11,12}$ to create integrated and spatially resolved SEDs in three radial bins at $|r| \lesssim 2 r_{e}$ for four, diverse, $z \sim 1.3$ galaxies selected for their brightness and spectral quality. Fitting these SEDs provides our SFHs (Section 3). We defer to Schmidt et al. (2014) and Treu et al. (2015) for GLASS' details, but review some relevant aspects here.

\subsection{GLASS Spectroscopy}

GLASS obtained slitless WFC3IR G102 and G141 grism spectra $(\lambda \sim 8800-16700 \AA)$ covering ten HFF/CLASH cluster-magnified sightlines. To mitigate against nearby object contamination, two spectra were taken per sightline at roughly orthogonal roll angles over $5(\mathrm{G} 102)+2(\mathrm{G} 141)$ orbits per orient, reaching similar depths in each grism. For the objects studied here, however, usable data come from only one orient (Section 2.2).

A modified 3D-HST pipeline (Brammer et al. 2012; Momcheva et al. 2016) associates these spectra with objects identified in deeper CLASH/HFF pre-imaging, and subtracts contaminating light from nearby traces. FITS cubes are produced for each source in each grism at each orient. We reference relevant layers of these in typewritten text to aid reproducibility (Appendix A provides a thorough discussion), but,

\footnotetext{
${ }^{10} \mathrm{https}$ ///archive.stsci.edu/prepds/glass/

${ }^{11} \mathrm{https}: / /$ archive.stsci.edu/prepds/clash/

${ }^{12}$ https://archive.stsci.edu/prepds/hff/
} 
at root, rely on each source's postage stamps, 2D spectra, and RMS and contamination maps. Section 2.3 details how these objects guide 1D spectral extraction.

The GLASS database contains 23265 objects. Of these, 3022 have $\mathrm{F} 140 \mathrm{~W}_{\text {GLASS }} \leq 24$ and so were inspected and assigned a fiducial spectroscopic redshift (zGLASS). All redshifts quoted here are rederived in the analysis (Section 3), but both estimates agree within uncertainties (Table 1).

\subsection{Sample Selection}

We aim to spectrophotometrically reconstruct spatially resolved SFHs to draw more meaningful longitudinal inferences from cross-sectional data. In scheme, we follow Kelson et al. (2014) and Dressler et al. (2016), Newman et al. (2014), and Domínguez Sánchez et al. (2016), who demonstrated the potential of this approach using similar but spatially integrated data at $z \sim 0.7, z \sim 1.8$, and our $z=1-1.5$ range, respectively.

The critical aspect of these data is that photometry samples the rest-UV/-IR where SFH information is low (current SFR and its integral, $M_{*}$, respectively). Meanwhile, spectroscopy covers the high information-density rest-optical, containing details on the intermediate age stellar populations that provide leverage on the path a galaxy took to reach those endpoints. "High" spectral resolution $\left(R_{\text {eff }} \gtrsim 70\right)$ is thus present where it is needed. This said, we diverge from the above studies in that Spitzer IRAC's larger PSF precludes us from using those data for spatially resolved photometry.

We stress that, for the case studies possible at this stage, data quality, not quantity, is key: While we will speculate on their broader implications (Sections 4.3, 5.1), we are not yet so interested in the representativeness of any one finding, but the potential of what can be learned from these data/methods. Given the critical role of spectroscopy in this exercise, the GLASS database determines sample selection.

From the 2176 sources at $1.0 \leq z \leq 1.8$-where $z$ is $z_{\text {GLASS }}$ or a photometric redshift (Brammer et al. 2008; Castellano et al. 2016; Morishita et al. 2017)—we draw 76 sources with $\mathrm{F} 140 \mathrm{~W}_{\mathrm{GLASS}} \leq 21.8$. This ensures important age-sensitive features lie in the grism bandpasses (the $4000 \AA$ break, the G4300 to Mgb continuum, as many Balmer lines as possible), avoids selecting against passive systems lacking a secure (emission line-based) $z_{\text {GLASS }}$, and ensures $\langle S / N\rangle \approx 10$ per $\sim 10$ pix resolution element in the (folded) OUTER extractions (Section 2.3, Figure 1). We then select 29 objects with at least one pair of grism spectra with $\leq 30 \%$ of OUTER pix contaminated at $\leq 30 \%$, where "contaminated" is the ratio of the CONTAM to MODEL FITS layers (Appendix A). This quality control cut is arbitrary, but balances sample size with avoiding continuum biases from contamination subtraction residuals. (No source has $\leq 10 \%$ of pix at $\leq 10 \%$ contamination.)

Finally, we discard three obvious mergers, 18 poorly sampled sources (1 pix wide extraction boxes, some of which are stars), one case of bad sky subtraction, two post factoidentified low- $z$ interlopers, and one source for which the lognormal SFH failed to converge (Section 3). Ongoing mergers may merit future study, but, to the extent possible (Section 5.2), we aim to infer in situ growth and so avoid spectral superpositions of objects with distinct SFHs.

This leaves a final sample of four sources. Fortuitously, they constitute a meaningfully diverse cohort of a (1) continuously starforming ("CSF"), (2) strongly starforming ("SSF"), (3) recently quenched/poststarburst ("PSB"), and (4) anciently passive galaxy ("PAS").

Figure 2 shows these sources and their integrated spec- trophotometry; Section 4.1, Tables 1 and 2 describe them in detail. While modest $(\langle\mu\rangle \simeq 1.7)$, none of the sample would meet $S / N$ requirements without lensing magnification.

\subsection{D Spectra}

We extract an optimal $S / N$ integrated spectrum and three, folded, radial spectra from each source. To mimic typical analyses, the former is used to derive total galaxy properties, the latter resolved ones. Summing the spatial extractions produces self-consistent results (Appendix C). Obtaining and analyzing these requires estimating $1 \mathrm{D}$ half-light radii, $r_{e}$, optimal extraction spatial weights, and line spread functions (LSFs) using the GLASS postage stamps (DIRECT_IMAGE; stacks of all WFC3IR CLASH/HFF pre-imaging).

To avoid including near neighbors whose spectra are removed by the pipeline, this requires some modeling. We collapse the central $20 \times 20$ pix $(\sim 10 \times 10 \mathrm{kpc})$ of each image along the spectral axis (to obtain the target's spatial profile), or spatial axis (to obtain the LSF from its morphology). We then fit Moffat functions to these profiles-which reach beyond bulges, but not to outer disks (Figure 2) - and extrapolate to extend them to the full 60 pix stamp size. The LSF is used in SED fitting (Section 3); the spatial profile defines the optimal extraction weights (Horne 1986), and $r_{e}$, on which the radial extractions are based.

Figure 3 shows each source's CLASH/HFF stamps, 1D $r_{e}$ and 2D half-light radii, 2D spectra, radial extraction zones, and corresponding 1D spectra. The radial bins are:

- INNER: $\left|r / r_{e}\right| \leq 0.4$;

- MIDDLE: $0.4<\left|r / r_{e}\right| \leq 1.0$;

- OUTER: $1.0<\left|r / r_{e}\right| \leq 2.0$;

where the MIDDLE and OUTER bins are reflected over the GLASS pipeline-output spectral centerline (YTRACE; galactocentric radius $r \equiv y-\mathrm{YTRACE}=0)$ and averaged before fitting. Bin widths are thus $0.8,2 \times 0.6$, and $2 \times 1.0 r_{e}$, respectively. While somewhat arbitrary and subject to pixelation, these were chosen to counter decreasing $S / N(r)$ while preserving

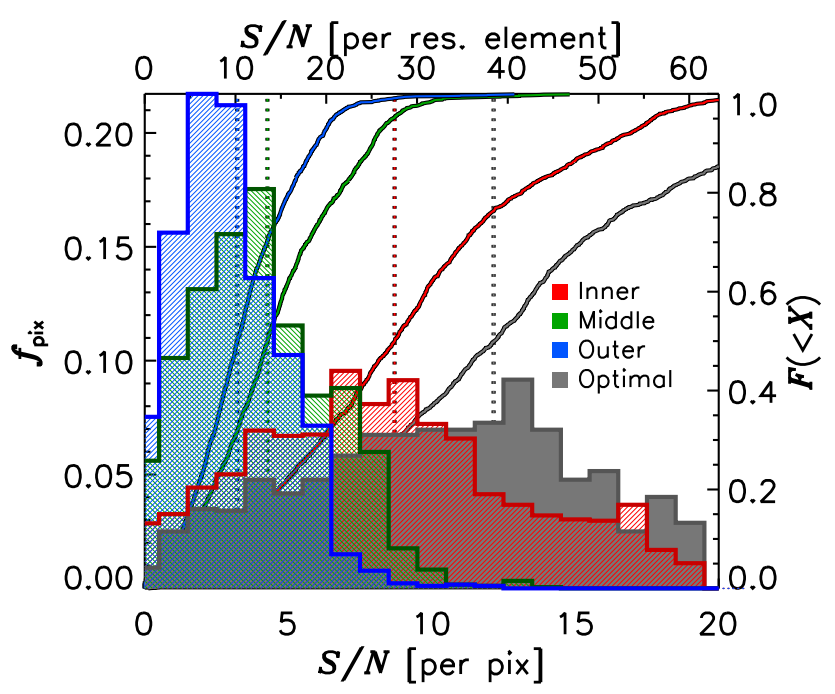

FIG. 1.- $S / N$ histograms and cumulative distributions for the 16 sample spectra (four per galaxy). INNER (red) covers $\left|r / r_{e}\right| \leqslant 0.4$; MIDDLE (green) and OUTER (blue) reach $\left|r / r_{e}\right| \in(0.4,1.0]$, and $\in(1,2]$, respectively. Optimal extractions are plotted in grey. Dotted vertical lines show median $S / N$. 
TABLE 1

BASIC SOURCE INFORMATION

\begin{tabular}{|c|c|c|c|c|c|c|c|c|c|}
\hline Sightline & GLASS ID_PA $^{a}$ & Tag & $\begin{array}{l}\mathrm{RA}\left[{ }^{\circ}\right] \\
(\mathrm{J} 2000)\end{array}$ & $\begin{array}{c}\mathrm{DEC}\left[{ }^{\circ}\right] \\
(\mathrm{J} 2000)\end{array}$ & $z^{\mathrm{b}}$ & $z_{\text {GLASS }}{ }^{c}$ & Magnification $(\mu)^{\mathrm{d}}$ & Pre-survey & $\begin{array}{c}\mathrm{F} 140 \mathrm{~W}_{\mathrm{GLASS}}^{\mathrm{e}} \\
(\mathrm{AB})\end{array}$ \\
\hline MACS0744 & $00660 \_2$ & PAS & 116.2281 & 39.46423 & $1.257 \pm 0.005$ & $1.260 \pm 0.006$ & $1.87 \pm 0.24$ & CLASH & 21.42 \\
\hline MACS 1149 & 00900_1 & SSF & 177.4089 & 22.40319 & $1.021 \pm 0.001$ & $1.033 \pm 0.007$ & $1.67 \pm 0.23$ & CLASH, HFF & 21.22 \\
\hline MACS1423 & 01916_2 & PSB & 215.9373 & 24.06136 & $1.423 \pm 0.002$ & $1.435 \pm 0.006$ & $1.52 \pm 0.24$ & CLASH & 21.34 \\
\hline MACS2129 & $00451 \_2$ & CSF & 322.3719 & -7.68199 & $1.367 \pm 0.001$ & $1.368 \pm 0.006$ & $1.66 \pm 0.24$ & CLASH & 21.72 \\
\hline
\end{tabular}

NotE. — a GLASS source number and orientation of the analyzed spectra; "_1” corresponds to the GLASS position angle closest to zero. b Basis of this analysis; from full spectrophotometric fitting (Section 3). ${ }^{\mathrm{c}}$ GLASS catalog redshift; error corresponds to a $\sim 10$ pix LSF FWHM at observed H $\alpha$; roughly $\sigma_{v}=2000 \mathrm{~km} \mathrm{~s}^{-1} .{ }^{\mathrm{d}}$ From the online calculator (HFF) or the maps of Zitrin et al. (CLASH; Section 2.5). ${ }^{\mathrm{e}}$ GLASS catalog SExtractor MAG_AUTO estimate.
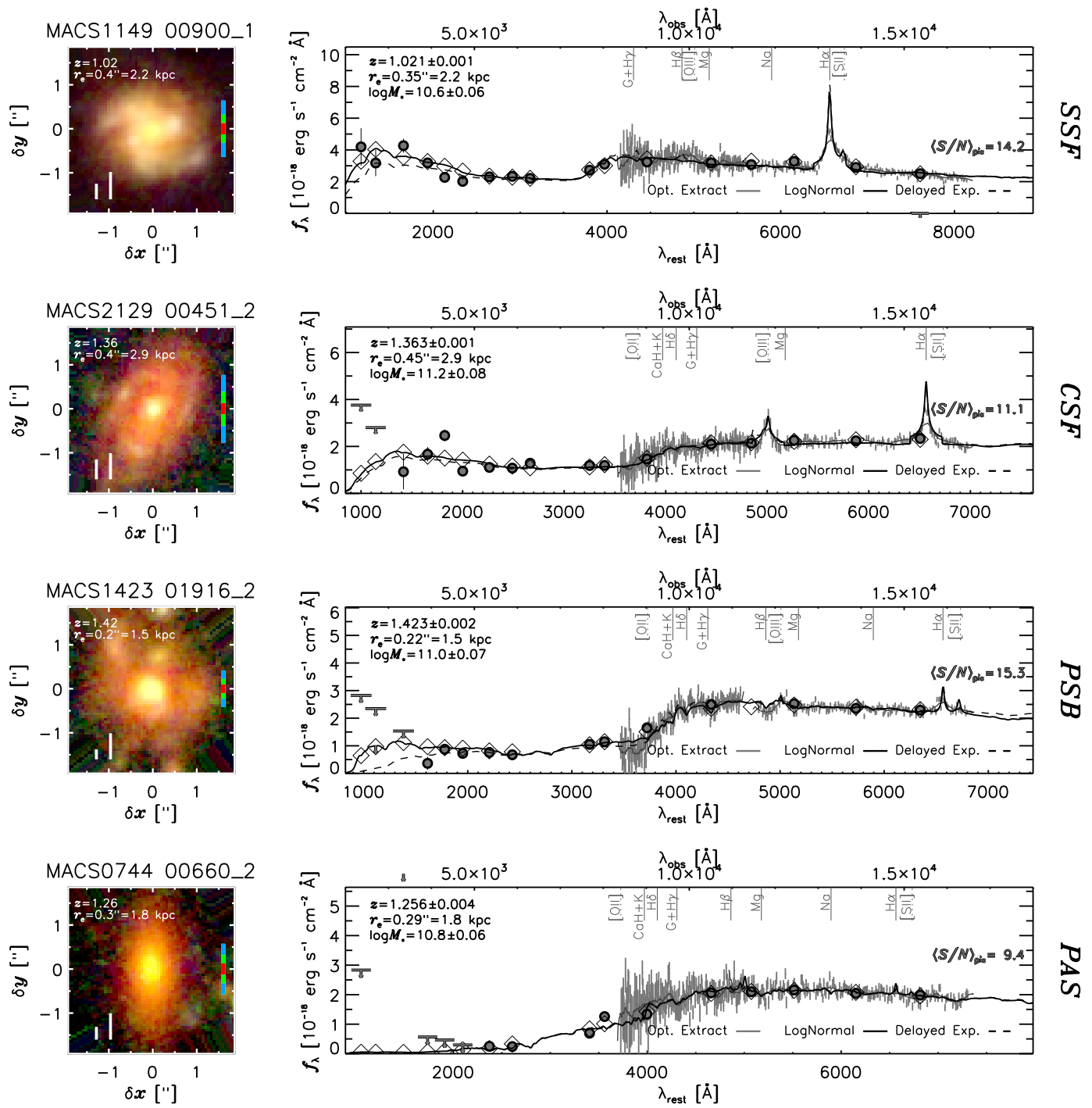

FIG. 2.- Color CLASH/HFF stamps and optimal GLASS spectra of the four sample objects (Table 1) ordered by decreasing sSFR from top. The smaller white scale-bar on each $\sim 4^{\prime \prime} \times 4^{\prime \prime}$ image shows $r_{e}$; the larger, $5 \mathrm{kpc}$ at the source redshift. Multicolored scale bars at right show the location/width of the radial spectral zones (Section 2.3) color-coded as in Figure 2 and all further plots. CLASH/HFF photometry/2 $\sigma$ limits are denoted by circles and arrows, respectively, with open diamonds showing the PYSPECFIT model values assuming lognormal (solid) or delayed exponential SFHs (dashed lines). Spectra are plotted as their $1 \sigma$ uncertainty interval at each $\lambda$. Numbers at the red ends of the spectra give mean per-pixel $S / N$. Other integrated physical parameters are listed at top-left.

spectral line shapes. Each zone's 1D spectrum is simply the mean of all unmasked pixels (SCI $>0$, CONTAM/SCI $<0.01$; Appendix A) in the CONTAM-subtracted 2D SCI spectrum at each $\lambda$. Uncertainties are the identical (quadrature) sums from the WHT RMS-map. GLASS 2D spectra are cut from interlaced-not drizzled-mosaics, so all pixels are independent. We find no significant backgrounds.

Note: as defined, " $r e$ " is not the half-light radius returned by, e.g., SExtractor or GALFIT (Bertin \& Arnouts 1996; Peng et al. 2002). Rather, it is the $y$ extent containing half the light 

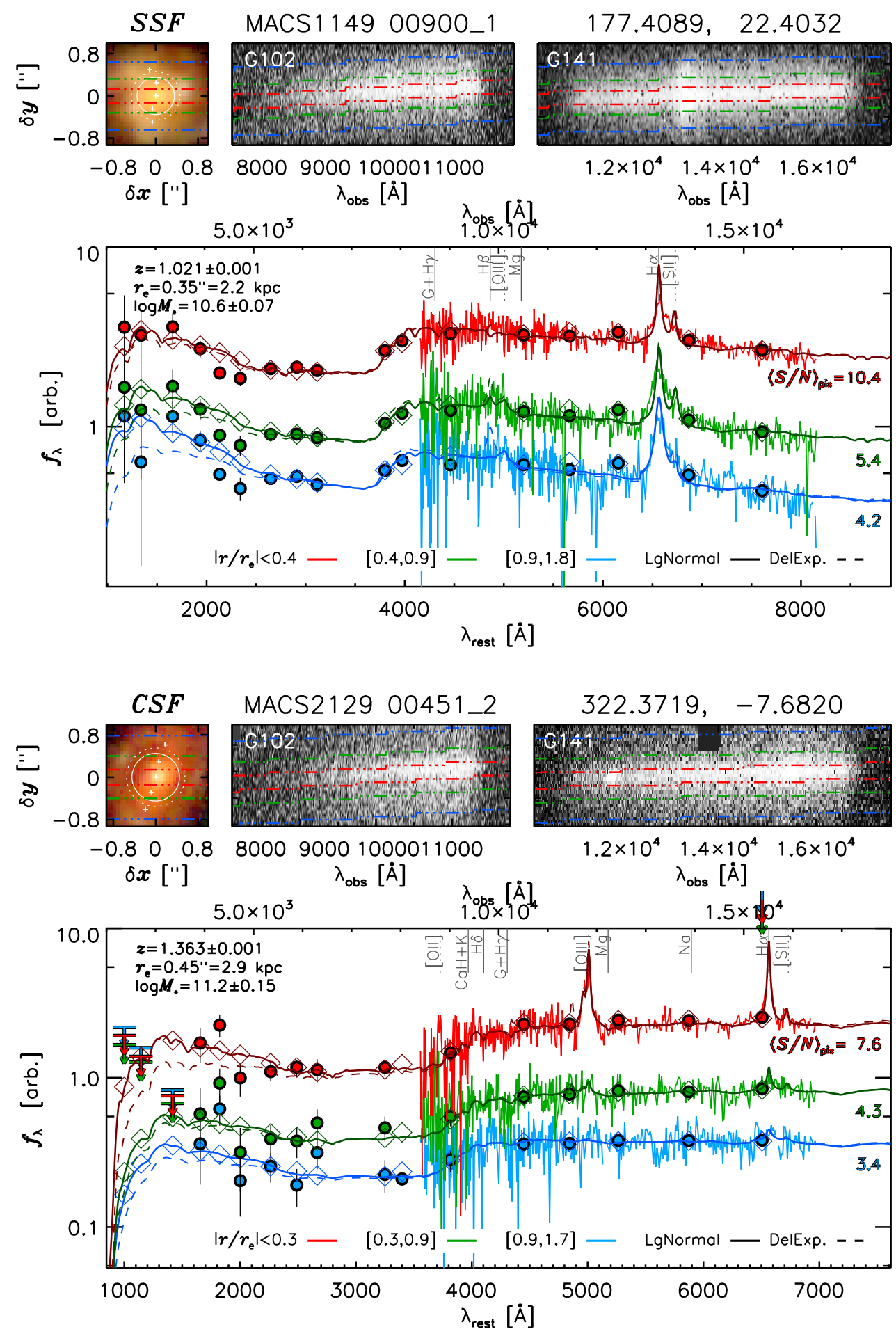

FIG. 3.- Images, 2D spectra, and radially resolved 1D extractions for SSF and CSF (others shown in the continuation of this plot). Top-left images are insets of those in Figure 2 showing 1D $r_{e}$ (solid) and 2D half-light radii (dashed circles), the extraction regions, and the light-weighted $(x, y)$ centroids for each. Rightward of these are the G102/141 2D GLASS spectra, with extractions zones overplotted in red (INNER), green (MIDDLE), and blue (OUTER). These are stepped as the spectra are not $(x, y)$ rectified. Black areas on the 2D spectra show masking due to contamination or missing data. The resulting 1D spectra are plotted in the main panel, color-coded by region, with CLASH+HFF photometry shown as in Figure 2. The best-fit PYSPECFIT lognormal (delayed exponential) SED fit is plotted as a solid (dashed) line, with open diamonds showing the predicted locations of the photometry. In all but PSB, the lognormal provides as good a fit to the data as the delayed exponential SFH. The radial extent of each extraction zone is listed at bottom.

collapsed across $x$. This is appropriate for defining spectral bins by spatial $S / N$, but underestimates $2 \mathrm{D}$ half-light radii. This effect is small except in ID 01916_2 ("PSB"; Figure 3).
Finally, we estimate LSFs independently in each zone. This accounts for meaningful galaxy chord profile variations and provides light-weighted bin centroids to assess position angle 

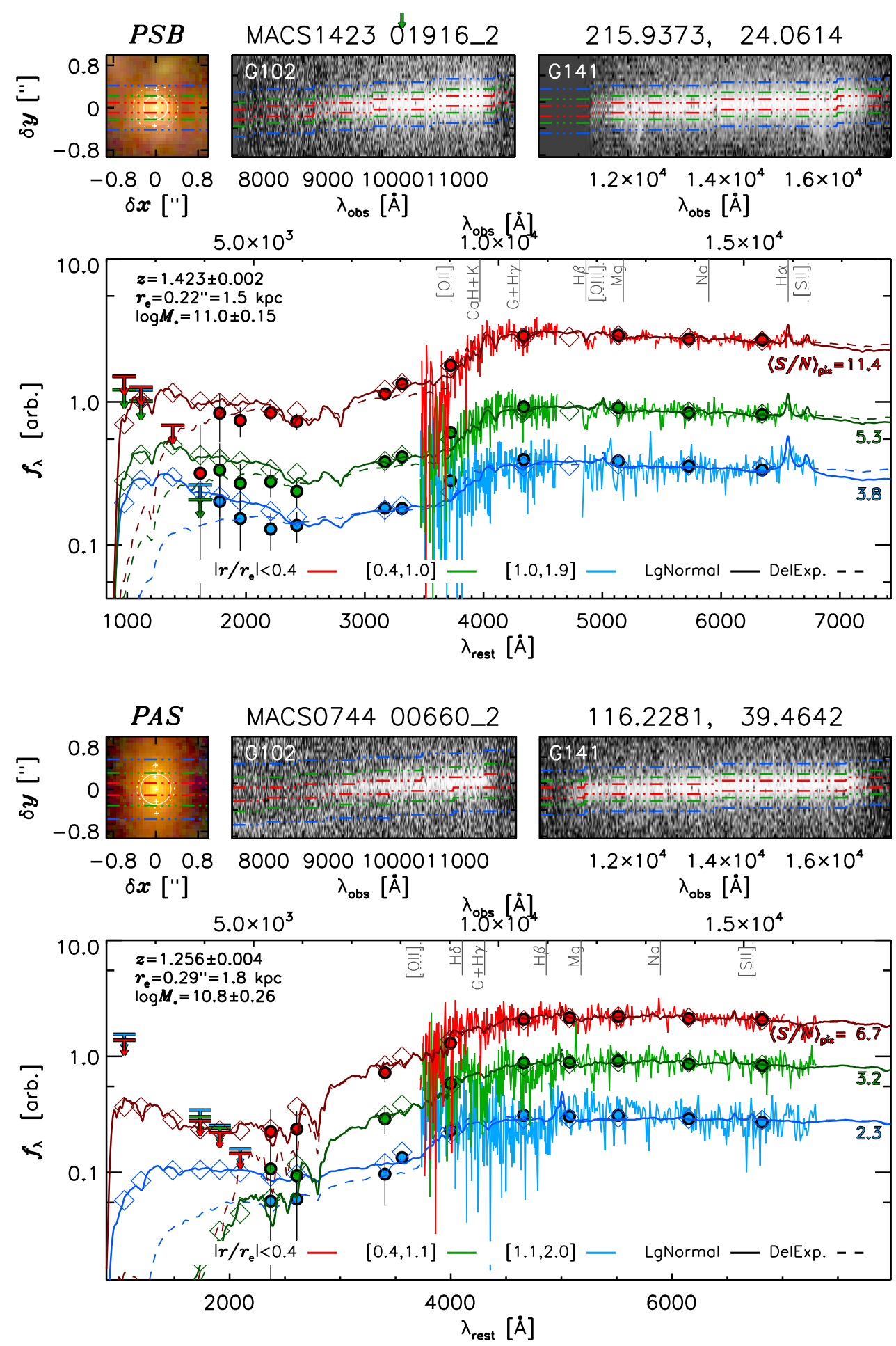

FIG. 3 (CONT'D).- Images, 2D spectra, and radial 1D spectra for PSB and PAS.

effects: At G102/141 spectral resolution, PAs near $45^{\circ} \mathrm{im}-$ pose a detectable geometric $r-\lambda$ covariance. Unlike typical (physical) $v-\lambda$ covariance at higher resolution, this is due to the slitless grisms reproducing an image of the entire source at every wavelength, not the doppler shift. Such offsets can reach $\sim 3$ pix (30\% LSF FWHM; $\sim 60 \AA ; \Delta z \sim 0.005$; Figures $3,16)$ and so should be accounted for in line analyses.

\subsection{Photometry}

Each source is covered by 17-band CLASH imaging, with one having deeper, supplementary data in the 7 HFF bands (Table 1). These reach into the rest-UV $(\sim 1500 \AA)$, providing critical leverage on dust and recent star formation.

We incorporate these data by cutting stamps/RMS maps for each source from its full, PSF-matched CLASH/HFF mo- 
saic (Morishita et al. 2017), resampling and rotating these to the GLASS plate scale/orientation (Appendix A). Resolved photometry is then the sum in each of radial zone from the cutouts. To match the spectra, INNER data is left as-is, while upper and lower MIDDLE and OUTER counts are averaged before measuring. For the optimal extraction, the zones are summed after weighting by the spatial profile.

Since all images are convolved a priori to F160W resolution, the resultant spectrophotometry is effectively aperturematched. Comparing synthetic F140W magnitudes from the GLASS spectra to broadband fluxes suggests an absolute accuracy of $\pm \sim 30 \%(1.5 \sigma)$. Yet, relative spectrophotometric agreement is more important in SED fitting. As we correct the photometry for galactic extinction (Schlafly \& Finkbeiner $2011),{ }^{13}$ we ultimately tie the spectra to those data by applying a single multiplicative constant. Figures 2 and 3 demonstrate that the subsequent agreement is excellent.

This process yields four, radially resolved, 17-band + G102/141 spectrophotometric datasets per galaxy ready for SPS modeling. Extant spatially integrated data not used in the modeling serve as cross-checks (Section 4.2).

\subsection{Lensing Magnification}

Lensing boosts apparent fluxes and sizes. Hence, estimating absolute quantities using those data $\left(M_{*}\right.$, SFR, intrinsic size) requires knowledge of a source's magnification, $\mu$. This is provided by models built for the GLASS cluster sightlines by a number of teams (Zitrin et al. 2009, 2013; Jauzac et al. 2014; Johnson et al. 2014; Richard et al. 2014; Grillo et al. 2015; Ishigaki et al. 2015; Wang et al. 2015; Hoag et al. 2016; Caminha et al. 2017). Since $\mu$ is applied to derived quantities, this step is independent of the preceding.

ID 00900_1 ("SSF") lies in the HFF footprint, so we download its default set of values from the public HFF lens models, ${ }^{14}$ discard the extremes, and adopt the mean (1.67) and scatter $(0.23 ; 14 \%)$ of the other inferences as $\mu$ and its error.

All other sources lack HFF lens models. For these, we average the "LTM-Gauss_v2" and "NFW_v2" Zitrin et al. (2009, 2013) models for $\mu$. For uncertainties, we add their $3 \sigma$ formal errors $(\lesssim 0.05)$ in quadrature with the (much larger) HFF model dispersion for SSF. Identical models are not available, but the "LTM-Gauss_v1" best estimate is consistent with SSF's HFF-derived $\mu$ at $2.4 \sigma$. In all cases, $\mu$ is a small correction ( $\lesssim 0.3$ dex; Table 1$)$, and ratios such as sSFR $\equiv$ $\mathrm{SFR} / M_{*}$ and $M_{*}$ or SFR surface densities are $\mu$-independent.

\section{SPECTRAL SYNTHESIS MODELING}

We fit the full spectrophotometric SED (broadband fluxes + spectra) to infer the sample galaxies' spatially integrated and resolved properties $-M_{*}$, SFR, $A_{V}$ for each optimal, INNER, MIDDLE, and OUTER dataset, marginalized over all other quantities - and SFHs based on delayed exponential and lognormal parameterizations. We adopt the lognormals for our main analysis since they are smooth over all $t$, but using both allows us to assess SFH systematics (Section 4.2) and check certain results (Appendices D, E).

We use PYSPECFIT for all fitting (Newman et al. 2014). This is a flexible, Bayesian python package wrapped around the multinest Monte Carlo Markov Chain engine (Feroz \& Hobson 2008). We assume Bruzual \& Charlot (2003) tem-

\footnotetext{
${ }^{13} \mathrm{https}: / /$ ned.ipac.caltech.edu/forms/calculator.html

${ }^{14}$ https://archive.stsci.edu/prepds/frontier/lensmodels/\#magcalc
}

plates, a Salpeter (1955) initial mass function, solar metallicity (Gallazzi et al. 2005, 2014), a Calzetti et al. (2000) dust law, and a $\chi^{2}$ likelihood function (see Newman et al. 2014; Eq. B1). Appendix B lists other fit parameters, which include $\mathrm{H} \alpha, \mathrm{H} \beta,[\mathrm{O} \mathrm{III}]$, and [S II] emission line equivalent widths.

The two SFH models are used in two fitting passes. First is the delayed exponential (e.g., Lee et al. 2010):

$$
\operatorname{SFR}(t) \propto \frac{t-t_{\text {start }}}{\tau_{\exp }^{2}} \exp \left[-\frac{t-t_{\text {start }}}{\tau_{\text {exp }}}\right],
$$

where $t$ is cosmic time, $\tau_{\exp }$ is an $e$-folding timescale, and $t_{\text {start }}$ is the beginning of the SFH. This last parameter is independent of $t$ (except that $t_{\text {start }} \leq t_{\text {obs }}$ ), so the SFH can be normalized without knowing its redshift. This is not true of the lognormal SFH (below). Hence, while Appendix D discusses $\tau_{\text {exp }}$ and "age" ( $\left.=t_{\text {obs }}-t_{\text {start }}\right)$ trends, the core purpose of this fit is fine redshift estimation, an input to step two.

Next, we fit a lognormal SFH (Gladders et al. 2013):

$$
\operatorname{SFR}(t) \propto \frac{1}{t \sqrt{2 \pi \tau^{2}}} \exp \left[-\frac{\left(\ln t-T_{0}\right)^{2}}{2 \tau^{2}}\right],
$$

where $T_{0}$ is the half-mass time (in $\ln \mathrm{Gyr}$ ) and $\tau$ controls its width. This form has significant advantages over the delayed exponential, including a smooth derivative over all $t$ avoiding unrealistic $d M_{*} / d t$ at late epochs (Figure 18)—and better agreement with SFHs from simulations (Diemer et al. 2017; Ciesla et al. 2017). Further, they reproduce many ensemble observations at $z \lesssim 8$ (Gladders et al. 2013; Abramson et al. 2015, 2016; Dressler et al. 2016). Hence, unless stated otherwise, this SFH is the default source for derived quantities. That said, the lognormal's lack of discontinuities and the fact that its decline is, by definition, slower than its rise make it a poor description in some cases (Diemer et al. 2017; Iyer \& Gawiser 2017; Section 4 below).

Since the lognormal has SFR $>0$ for all $t$, normalizing it to a total $M_{*}$ requires knowing a source's redshift (the upper bound to $\int \mathrm{SFR} d t$ ). Hence, all lognormal fitting is fixed to the redshift measured using the delayed exponential SFH.

SED fitting thus produces a redshift, and $4 \times 2$ sets of $M_{*}+\mathrm{SFR}+\mathrm{SFH}$ parameters for each galaxy describing the optimal, INNER, MIDDLE, and OUTER extractions given delayed exponential or lognormal SFHs. Mass estimates are consistent, though SFRs may not be (Figure 5; Section 4.2). Broad priors are placed on the SFH parameters and other outputs (e.g., emission line equivalent widths and $A_{V}$ ). Best-fit values are the medians of the resultant multinest posterior distributions, with the 16th-84th percentiles as formal uncertainties. Full parameter covariances are also output and used when necessary-e.g., when reconstructing the SFHs themselves.

\section{RESULTS}

In this section, after characterizing the sample's spatially integrated (Section 4.1) and resolved (4.2) diversity at $t_{\mathrm{obs}}$, we use the lognormal SFHs to chart each galaxies' development over multiple epochs (4.3). Projecting these systems back in time reveals many signs of inside-out (bulge-first) growth, hints of a link between high bulge mass fractions and non-constant SFHs, and a difference between longitudinal and cross-sectional size reconstructions (4.3.2), encouraging further study of their relationship.

All quantities are magnification-corrected and incorporate that uncertainty. 
TABLE 2

DERIVED INTEGRATED PROPERTIES FROM OPTIMAL EXTRACTIONS

\begin{tabular}{|c|c|c|c|c|c|c|c|}
\hline Tag & $\log M_{*} / \mathrm{M}_{\odot}^{\mathrm{a}, \mathrm{b}}$ & $\log \mathrm{SFR} / \mathrm{M}_{\odot} \mathrm{yr}^{-1 \mathrm{a}, \mathrm{b}}$ & $r_{e}[\mathrm{kpc}]^{\mathrm{b}}$ & $r_{e, 2 \mathrm{D}}[\mathrm{kpc}]^{\mathrm{b}, \mathrm{c}}$ & $A_{V}[\mathrm{mag}]^{\mathrm{a}}$ & $\left(T_{0}, \tau\right)[\ln \mathrm{Gyr}]^{\mathrm{a}}$ & $\left(\right.$ age,$\left.\tau_{\text {exp }}\right)[\mathrm{Gyr}]$ \\
\hline PAS & $10.83 \pm 0.06(0.17)$ & $<-1.18^{\mathrm{d}}$ & $.76 \pm 0.11$ & $2.15 \pm 0.13$ & $0.09 \pm 0.09$ & $(1.03 \pm$ & $(1.4$ \\
\hline SSF & $10.57 \pm 0.06(0.06)$ & $1.40 \pm 0.06(0.12)$ & $2.20 \pm 0.14$ & $2.86 \pm 0.19$ & $1.06 \pm 0.02$ & $(1.64 \pm 0.00,0.10 \pm 0.00)$ & $(0.70 \pm 0.09,0.24 \pm 0.04)$ \\
\hline PSB & $10.95 \pm 0.07(0.12)$ & $0.54 \pm 0.10(0.93)$ & $1.54 \pm 0.11$ & $2.88 \pm 0.21$ & $0.41 \pm 0.05$ & $(1.21 \pm 0.01,0.10 \pm 0.00)$ & $(0.42 \pm 0.09,0.06 \pm 0.02)$ \\
\hline CSF & $11.15 \pm 0.07(0.10)$ & $1.59 \pm 0.11(0.39)$ & $2.94 \pm 0.20$ & $3.61 \pm 0.24$ & $1.19 \pm 0.12$ & $(1.32 \pm 0.35,0.68 \pm 0.31)$ & $(3.51 \pm 0.66,1.42 \pm 0.60)$ \\
\hline
\end{tabular}

NOTE. - ${ }^{\mathrm{a}}$ Lognormal SFHs; parentheses show summed INNER + 2 MIDDLE + 2 OUTER $M_{*}$ or SFR errors; delayed exponential $M_{*}$ but not necessarily SFR are consistent (Figure 5, Table 4). ${ }^{\mathrm{b}}$ Magnification-corrected (Table 1 ); uncertainties incorporate $\mu$ errors. ${ }^{\mathrm{c}}$ SExtractor half-light radius. ${ }^{\mathrm{d}} 2 \sigma$ limit.

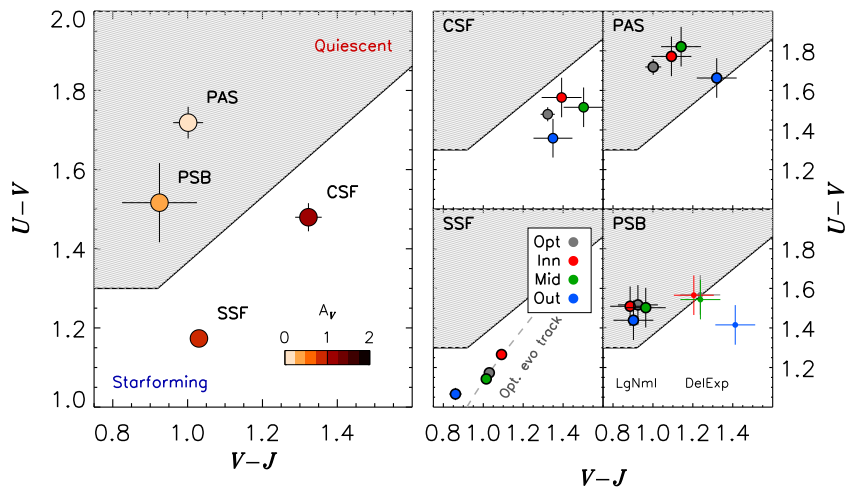

FIG. 4.- Left: Integrated synthetic rest- $U V J$ colors from the best-fit SFHs coded by $A_{V}$. Consistent with their appearances (Figure 2), CSF and SSF lie in the diagram's starforming region and are globally more extinguished than their quiescent counterparts (Figure 8). Right: resolved colors from the radial spectral extractions. CSF and SSF have $x$ or $y$ gradients such that INNER regions are redder than OUTER ones. The quiescent PAS and PSB show opposite/no gradients. Notably, SSF's pieces lie roughly along the trajectory of its integrated SED fit, suggesting larger- $r$ regions developmentally lag INNER ones ("inside-out growth"; Section 4.3.2). As we lack resolved rest-J coverage, PSB's truncated history may drive SFH systematics to which the inferred colors are sensitive (Section 4.2).

\subsection{Integrated Properties at $t_{\mathrm{obs}}$ \\ 4.1.1. Masses and Star Formation Classes}

In terms of monolithic properties (Table 2), the sample spans $10.5 \lesssim \log M_{*} \lesssim 11.2$ and a range of SF states- $-0.1 \lesssim$ $\mathrm{SFR} / \mathrm{M}_{\odot} \mathrm{yr}^{-1} \lesssim 40$; i.e., likely progenitors of $z=0$ Milky Way (MW) to $\geq$ M31-mass galaxies (see below).

Figure 2 shows the sample's integrated spectrophotometry. Notable $\mathrm{H} \alpha$ and UV flux combined with prominent disks/blue spiral arms reveal IDs 00451_2 and 00900_1 to be starforming. The other two sources-IDs 00660_2 and 01916_2-show either no or much less H $\alpha / \mathrm{UV}$ flux, whichcombined with their spheroidal, redder appearance-reveal them to be passive. The sources' positions in the UVJ (Figure 4, left; Williams et al. 2009) and SFR- $M_{*}$ planes (Figure 5) reflect these statements: 00451_2 and 00900_1 lie outside the quiescent box and on the $z \sim 1.25$ SF "Main Sequence" (SFMS; Noeske et al. 2007; Whitaker et al. 2014); 00660_2 and 01916_2 lie in the box and off the SFMS.

While evenly split between starforming and passive galaxies, Figure 2's spectra expose more sample diversity. First, despite being "normal” SFMS galaxies, 00451_2 and 00900_1 are quite distinct: the former is redder and has a shallower UV slope than the latter, suggesting it is more mature/farther from its peak SFR (Section 4.3). This is consistent with 00451_2's $\sim 4 \times$ greater mass, reduced $\mathrm{EW}(\mathrm{H} \alpha+[\mathrm{N} \mathrm{II}])(\sim \mathrm{sSFR})$, and the fact that its resolved components actually lie off the SFMS

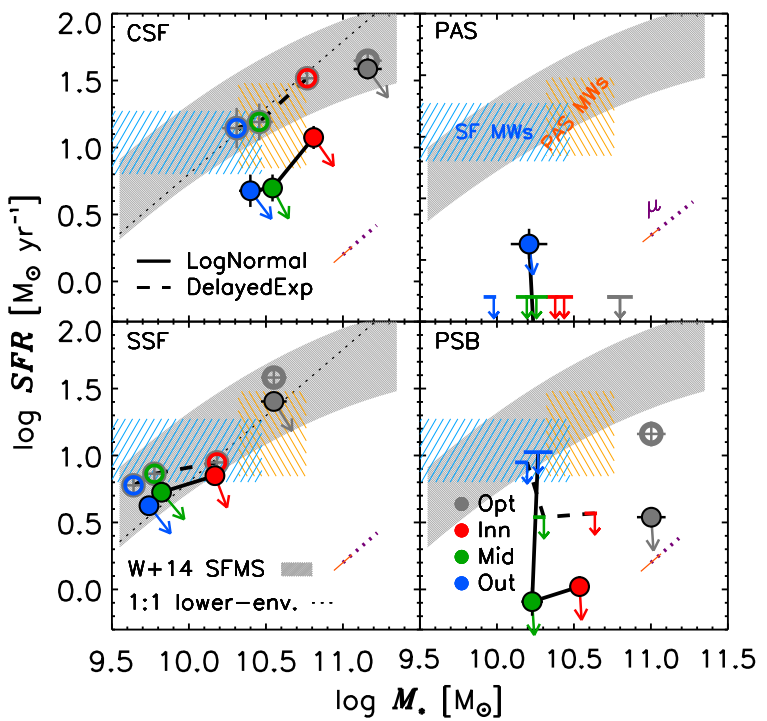

FIG. 5.- The spatially resolved SFR- $M_{*}$ plane. Starforming (passive) systems are plotted at left (right). Solid symbols/filled lines show lognormal results; open symbols/dashed lines delayed exponentials. Bars denote $2 \sigma$ upper limits; arrows $\left(M_{*}, \mathrm{SFR}\right)(t)$ unit vectors averaged over $t_{\mathrm{obs}} \pm 1 \mathrm{Gyr}$. Grey shading shows the $z \sim 1.25$ SFMS (Whitaker et al. 2014); blue/orange blocks locate progenitors of $z=0 \mathrm{MW}$-mass starforming and passive galaxies (Gladders et al. 2013). Resolved and integrated metrics (colored/grey circles, respectively) support similar inferences with telling exceptions: The SFMS' bend allows galaxies to be on the locus while their components are not (CSF), suggesting SFMS-based inferences can yield biased insights into SF physics. Dotted purple/thin orange lines show magnification effects/uncertainties.

(Section 4.2; the locus' bend keeps it defined as "starforming"). Thus, we refer to these starforming systems as:

$$
\begin{aligned}
& \text { - 00451_2 } \equiv \text { “CSF”- Continuously Starforming; } \\
& \text { - 00900_1 三 "SSF” — Strongly Starforming. }
\end{aligned}
$$

Notable [O III] emission suggests CSF hosts an AGN, confirmed by its resolved spectra and mass-excitation diagram (Juneau et al. 2014; Figures 3, 7).

The two passive galaxies are also distinct: 00660_2 is unquestionably old, with a red continuum that rises from the essentially absent UV to [O III], and strong G-band absorption. Meanwhile, 01916_2 has a bluer, A star-like spectrum (similar continuum near [O III] and $\mathrm{H} \gamma$; weak G-Band; Figures 4, 9), and higher UV flux. Combined with residual $\mathrm{H} \alpha+[\mathrm{N}$ II $]$ emission and the photometry's preference for the more rapidly decaying delayed exponential SFH (Section 4.2), this suggests 01916_2 was rapidly quenched within $\sim 1$ Gyr of $t_{\text {obs }}$ (Table 2; Dressler \& Gunn 1983; Poggianti et al. 1999). We therefore refer to these quiescent systems as: 
• 00660_2 $\equiv$ "PAS" — Passive;

- 01916_2 三 "PSB" — Post Starburst.

From Figure 5, SSF and PAS appear set to become $\sim$ MWmass $z=0 \mathrm{E} / \mathrm{S} 0$ s, while CSF and PSB are modern $\geq \mathrm{M} 31$ mass progenitors (see also Section 5.1.3). Given their aforementioned spectral diversity, dissecting these galaxies in space and time thus provides insights relevant to-if not representative of-important parts of parameter space.

Note: Given their consistency, we occasionally plot $M_{*}$ averaged over SFH choice (Figure 6). However, by default, SFRs reflect lognormal inferences. This (1) ensures selfconsistency with evolutionary projections (Section 4.3); (2) captures representative mass growth rates (which presumably change on timescales longer than the $\sim 10^{7} \mathrm{yr}$ on which $\mathrm{H}$ II regions evolve); (3) avoids line flux and dust correction biases due to $\mathrm{H} \alpha+[\mathrm{N} \mathrm{II}]$ and $\mathrm{H} \beta+[\mathrm{O}$ III $]$ blending (but see Wang et al. 2017); and (4) reduces ambiguities associated with active galactic nuclei (AGN), present in CSF. Nevertheless, using dust-uncorrected $\mathrm{EW}(\mathrm{H} \alpha+[\mathrm{N} \mathrm{II}])$ as an sSFR proxy yields similar trends to those inferred from full SED fitting (Appendix E, Figure 19).

\subsubsection{Size and Structure}

Figure 6, left, shows the sample on the size-mass plane. Considering their $2 \mathrm{D}$ half-light radii $(\sim 2.1-3.6 \mathrm{kpc})$, the passive PAS and PSB lie where they are expected to, while the starforming galaxies are small for their mass (van der Wel et al. 2014, though note we plot SExtractor and not GALFIT estimates). This could be due to chance or our selection criteria, as small galaxies' narrow spectra are less likely to be contaminated (Section 2.2). Trends hold in 1D $\left(r_{e} \sim 1.5-3.0 \mathrm{kpc}\right)$ except PSB shrinks notably (see also Figure 3 ).

Structure should also encode important evolutionary physics. Figure 6, right, shows the sample's mass-massconcentration- $M_{*}(r \leq 2.5 \mathrm{kpc}) / M_{*}$-relation. The systems span $\sim 30-60 \%$ in this quantity, which-to the extent that it reflects bulge-to-total $(B / T)$ ratios - places most on the $z=0$ 2 expectation (Lang et al. 2014; Abramson et al. 2014, latter plotted). CSF is, however, in the lower quartile for its mass, which may be revealing (Sections 4.3.2, 5.1).

We calculate mass concentrations by interpolating the stellar mass surface density in each radial zone $\left(\Sigma_{M_{*}}(r)\right)$ onto a finer grid, assuming circular symmetry, and integrating to $2 r_{e}$, e.g., $M_{*}$,int $(<r)=2 \pi \int_{0}^{r} r^{\prime} \Sigma_{M_{*}}\left(r^{\prime}\right) d r^{\prime}$. We force the outermost $r=2 r_{e}$ bin to match the optimal extraction $M_{*}$ estimates (Table 2). While not formally self-consistent, this can be repeated as we evolve the galaxies to different epochs to estimate half-mass radii and " $B / T$ " trajectories in a way comparable to cross-sectional studies (Morishita et al. 2015). Before re-normalizing, we obtain consistent total masses for two systems (CSF, PAS) with the others $\sim 0.3-0.4$ dex low, perhaps due to their clumpier structure (Figure 2). SFH systematics are $\Delta B / T \sim 0.1$, comparable to offsets assuming $B / T$ is the ratio of the F160W flux at $r<2.5 \mathrm{kpc}$ to SExtractor's FLUX_AUTO. As such, Figure 6, right, is most robustly interpreted to mean that these galaxies have normal bulge fractions for their mass except CSF, which is unexpectedly disky.

\subsection{Spatially Resolved Properties at $t_{\mathrm{obs}}$ : Colors, Mass and SFR Densities, and Dust}

Figure 4, right, shows color gradients in $U-V, V-J$, or both in the starforming systems CSF and SSF, such that

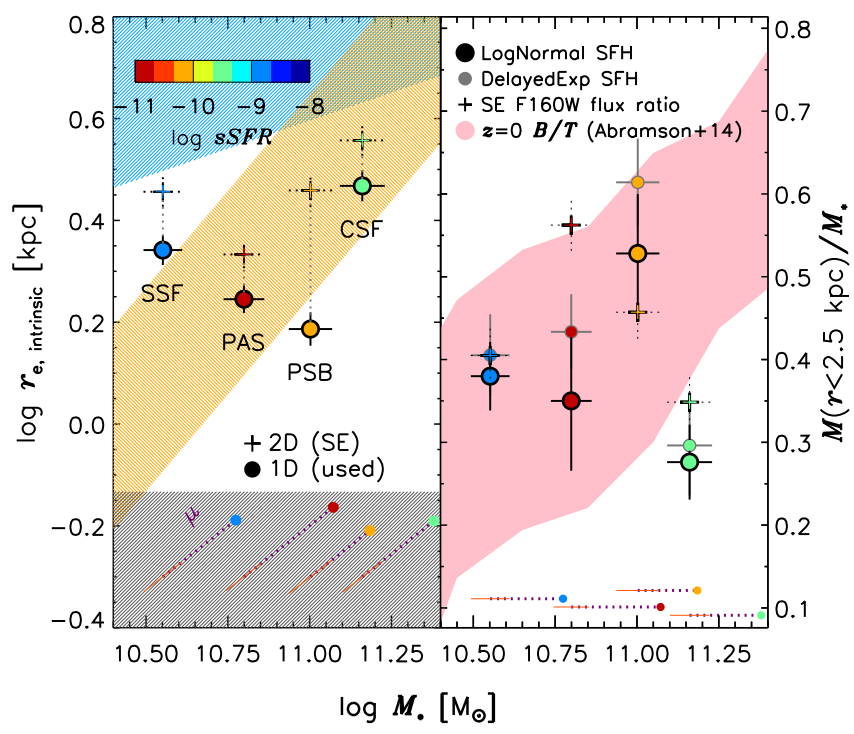

FIG. 6.- Left: the size-mass plane. Points are color-coded by sSFR with circles and crosses showing 1D $r_{e}$ used in this analysis and 2D SExtractor half-light radii, respectively. Blue and orange shading shows van der Wel et al. (2014)'s starforming and passive $z=1.25$ relations, with the HST resolution limit in black. Right: the $M_{*}(r<2.5 \mathrm{kpc})$ fraction $(\sim B / T)$-mass plane. Black/grey circles show lognormal/delayed exponential results, with Abramson et al. (2014)'s $z=0,25 \%-75 \%$ range shaded pink (a good description at all $z \lesssim 2$; Lang et al. 2014). Each galaxy is plotted at the mean of its two (consistent) total $M_{*}$ estimates (Figure 5, Table 4). All galaxies have normal $B / T$ for their mass except CSF, which is unexpectedly disk-dominated.

INNER regions are at least redder than OUTER ones. These trends are due to each zone's intrinsic stellar populations, not dust (Figure 8, bottom). This is expected (bulges are old) but it is interesting that SSF's zones fall roughly along the time trajectory of a single lognormal SFH (dashed line); i.e., the MIDDLE and OUTER regions simply lag INNER by some delay. We revisit this below and in Section 4.3.1.

The passive systems-PAS, PSB - are either uniformly red, or have gradients in the opposite sense, reddening with increasing $r$ at least in $V-J$. This may be due to dust (Figure 8 , bottom), or may reflect SFH systematics. These are significant in PSB, where the lognormal and delayed exponential models disagree at rest- $J$. Because delayed exponentials can fall faster than the lognormal, better mimicking what in reality may be a discontinuity, these fit PSB's UV photometry better at all $r$ (Figure 3). Regardless, the exponential UV fit quality and the lognormal color inferences agree in implying that whatever quenched PSB acted quasi-globally, such that much of the galaxy shut down at about the same time.

While it confirms the global nature of PSB's SFH truncation, Figure 3's radially resolved spectra conversely reveal the fact that the MIDDLE and OUTER regions of CSF show no signs of the AGN activity inferred from its integrated data (Figure 2; see also Figure 7). Further, their SFRs remain $\sim 3-$ $6 \mathrm{M}_{\odot} \mathrm{yr}^{-1}$, respectively.

That said, Figure 5 does suggest that these SFRs are below average for objects with CSF's INNER, MIDDLE, or OUTER regions' masses assuming lognormal SFHs (if not delayed exponentials; see below). Indeed, these components lie 1.5-2 $\sigma$ below the SFMS even though CSF as a whole appears normal. This is due to the bend in the SFMS at $\log M_{*} \gtrsim 10$ (e.g., Salim et al. 2007; Whitaker et al. 2012, 2014; Abramson et al. 2014; Schreiber et al. 2016) and affects the bluer SSF to a lesser ex- 


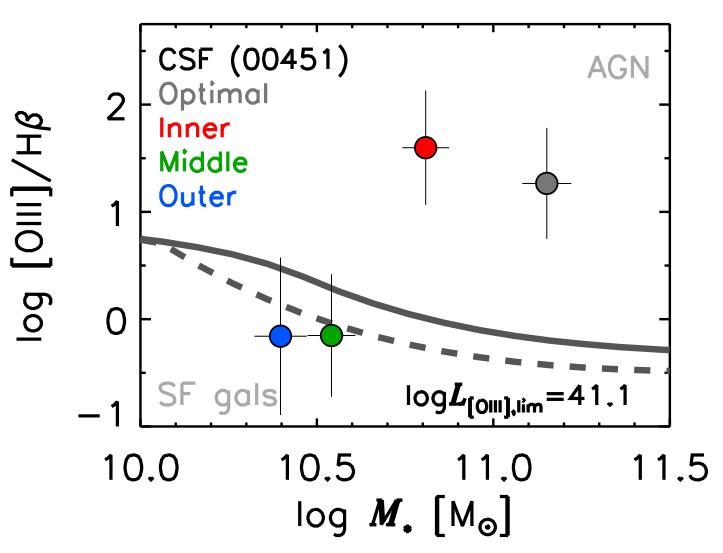

FIG. 7.- CSF's resolved mass-excitation diagram (Juneau et al. 2014) at GLASS' $2 \sigma F_{\mathrm{lim}} \simeq 2 \times 10^{-17} \mathrm{erg} \mathrm{s}^{-1} \mathrm{~cm}^{-2}$ (Schmidt et al. 2014, i.e., $\left.\log \left(L_{[\mathrm{OIII}]} / \mathrm{erg} \mathrm{s}^{-1}\right)_{\lim }=41.1\right)$. INNER/optimal estimates imply AGN activity (above the solid line). Meanwhile, MIDDLE and OUTER regions lie with normal starforming galaxies (below the dashed line). These maintain a combined $\mathrm{SFR} \sim 10-25 \mathrm{M}_{\odot} \mathrm{yr}^{-1}$, apparently unquenched by the AGN (Figure 5).

tent. Regardless, this_-and similar but opposite phenomena in $z \approx 0$ "Green Valley" systems (Dressler \& Abramson 2015; Vulcani et al. 2015a)-highlights key complications in inferring physics from the SFMS: It is hard to see how CSF could be normally starforming while its pieces are quenched. As now seen with emission lines (Sanders et al. 2017), integrated inferences are not always the sum of their parts, a dichotomy requiring spatially resolved data to understand.

Figure 8 quantifies further radially resolved sample trends. The top row shows the galaxies' surface mass density profiles: $\Sigma_{M_{*}}(r) \equiv M_{*}(r) / \operatorname{Area}(r)$, where $\operatorname{Area}(r)$ is based on the number of $0 . " 065 \times 0$."065 pixels in the SExtractor segmentation map in each radial zone. The left panel shows $\Sigma_{M_{*}}$ in absolute units, the right normalized to inner $1 \mathrm{kpc}$ means. Interestingly, the starforming CSF and SSF have steeper $\Sigma_{M_{*}}$ profiles compared to their passive counterparts (though SSF is the least dense system). This remains true, if less significant, when viewed in terms of physical radii instead of $r / r_{e}$ (Figure 19) and suggests either that (1) the passive systems have already undergone some amount of, e.g., minor-mergerdriven envelope building (e.g., Newman et al. 2012; Nipoti et al. 2012; Morishita \& Ichikawa 2016), or (2) the starforming systems are growing "inside-out," with their disks following their bulges (e.g., Eggen et al. 1962; Silk \& Norman 1981; Kepner 1999; Tacchella et al. 2015).

Figure 8, middle, provides some leverage on the latter issue. Instead of stellar mass, here we plot SFR surface density $-\Sigma_{\mathrm{SFR}}(r) \equiv \operatorname{SFR}(r) / \operatorname{Area}(r)($ left $)$ —and SSFR gradients (right). CSF exhibits a fairly constant $\operatorname{sSFR}(r)$, while SSF's rises by $\sim 40 \%-80 \%$ with increasing $r$; i.e., its center is perhaps half as active as its disk (see also Nelson et al. 2016b; Abdurro'uf \& Akiyama 2017). This is consistent with SSF's $U V J$ color gradient suggesting its bulge is relatively developmentally advanced. However, this is not true of CSF, whose star formation appears more uniform, suggesting its higher mass was built up more gradually at all $r$ (Section 4.3.2). Hence, these starforming galaxies had quite different pasts.

Nonetheless, CSF and SSF do share an interesting trait: While their OUTER sSFRs differ by $\gtrsim 3 \times$, they correspond to precisely the same SFR density $-\Sigma_{\mathrm{SFR}} / \mathrm{M}_{\odot} \mathrm{kpc}^{-2} \simeq 0.1-$
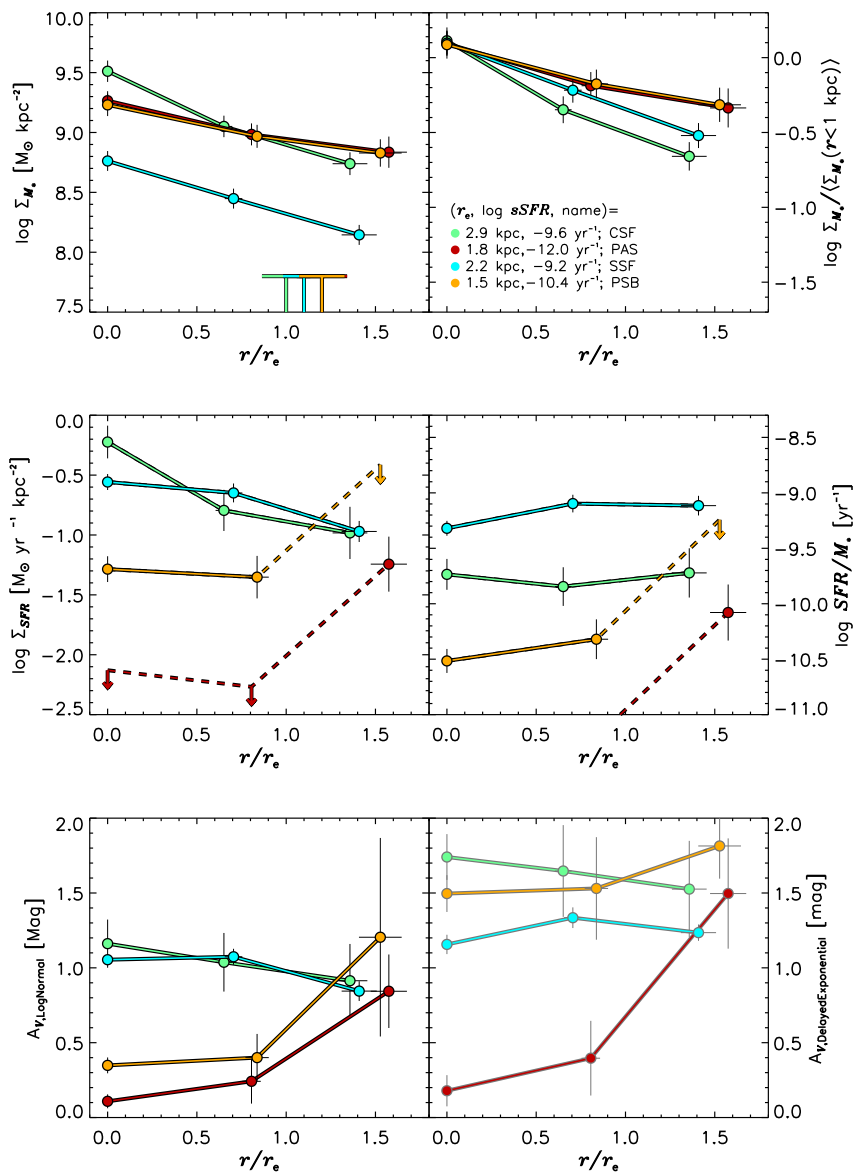

FIG. 8.- Top: Lognormal absolute (left) and relative (right) mass surface density $\left(\Sigma_{M_{*}}\right)$ profiles. The latter is normalized to the mean in each source's central kpc. Middle: Lognormal SFR surface density $\left(\Sigma_{\mathrm{SFR}}\right)$ and SSFR profiles (areas cancel). Bottom: $A_{V}$ (dust) profiles from lognormal (left) and delayed exponential SFHs (right). While the outermost sSFRs of the sample differ by $>10 \times$, they have consistent $\Sigma_{\mathrm{SFR}}$ at those radii (and all $r$ for the starforming systems), favoring, e.g., gas surface density over mass as an SFR governor. Note also the significant systematic offsets in the $A_{V}$ inferred using the two SFHs. This may point to physically meaningful, testable differences between the two SFHs (Section 4.2). Bin $x$-offsets reflect pixelation effects.

and indeed nearly identical SFRs (Figure 5). Taken together, Figure 8's middle panels imply that quantities beyond stellar mass are important to characterizing or controlling star formation in starforming galaxies: CSF's relatively heavy disk supports the same absolute level of star formation as SSF's lighter-yet-similarly sized disk. Without resolved data, this phenomenon would not have been detected.

This signal-where sSFR but not $\Sigma_{\text {SFR }}$ declines at fixed- $r$ with increasing mass-is not inconsistent with Nelson et al. (2016b)'s stacked HST H $\alpha$ map results (see their Figures 12, $13)$, though sample size prevents us from saying more.

Finally, Figure 8, bottom, shows lognormal- and delayedexponential-inferred $A_{V}$ trends (left/right, respectively). At left, the globally higher dust content inferred from CSF's and SSF's integrated extractions hold at all $r$ probed, in qualitative agreement with gas-phase Balmer decriment estimates by Nelson et al. 2016a. Yet, using either SFH, we infer that PAS, while clear of dust at $r \lesssim r_{e}$, has comparable $A_{V} \sim 1$ mag to the starforming galaxies at $\sim 1.5 r_{e}$. This trend is opposite to that inferred for a more massive $z \sim 2.15$ system by Toft et al. (2017), but could be a hint of ancient spiral arms and thus a 
TABLE 3

ID 01916_2 GLASS/MOSFIRE SPECTRAL INDEX COMPARISONS

\begin{tabular}{|c|c|c|c|}
\hline Feature $^{\mathrm{a}}$ & EW $_{\text {GLASS inf }}^{\text {Del. Exp. }}[\AA]$ & $\mathrm{EW}_{\text {GLASS inf }}^{\text {Lognormal }}[\AA]$ & $\mathrm{EW}_{\text {MOSFIRE }}[\AA]^{\mathrm{b}}$ \\
\hline $\mathrm{H} \delta_{A}$ & 9.3 & 7.4 & $5.3 \pm 1.1$ \\
\hline $\mathrm{H} \delta_{F}$ & 6.4 & 5.1 & $4.7 \pm 0.8$ \\
\hline $\mathrm{H} \gamma_{A}$ & 6.2 & 4.8 & $1.2 \pm 1.0$ \\
\hline $\mathrm{H} \gamma_{F}$ & 4.8 & 4.5 & $2.7 \pm 0.7$ \\
\hline G4300 & -0.5 & 1.2 & $2.7 \pm 1.1$ \\
\hline $\begin{array}{l}\text { NOTE. } \\
\text { (http://astro } \\
\text { uncertainti }\end{array}$ & pectra in $\mathrm{Fi}$ & $\begin{array}{c}{ }^{\mathrm{a}} \text { Lick } \\
\text { 1/index.table.h }\end{array}$ & $\begin{array}{lr}\text { IDS } & \text { definition } \\
\text { l). } & { }^{b} \text { Bootstrappe }\end{array}$ \\
\hline
\end{tabular}

sign of inside-out growth/quenching (as those authors' sSFR inference suggest), or conversely the contents of recently accreted satellites. Both scenarios are supported by the delayed exponential-derived age gradients, where PAS' OUTER region appears $\sim 1.5$ Gyr younger than its INNER one (Figure 18, top; also similar to Toft et al. 2017's findings).

The remaining system-PSB-presents other challenges. The lognormal fits imply a centrally clean $A_{V}$ trend similar to the older PAS. However, the delayed exponentials imply a flatter, $\sim 1$ mag more extinguished profile similar to the starforming systems'. Indeed, for all but PAS, delayed exponential $A_{V}$ are systematically higher than lognormal inferences.

This has to do with the geometry of the SFHs, specifically the exponential's delay: By definition, the model uses less than the maximal amount of time to produce the $M_{*}$ required to match a system's observed flux. As such, the delayed exponentials' mean $\operatorname{SFR}(t)$ are, by construction, higher than the lognormals', which start at $t=0$. This naturally leads to higher $\operatorname{SFR}\left(t_{\mathrm{obs}}\right)$ (Figure 5). Of course, despite the requisitely high SFRs, the delayed exponentials cannot simultaneously overshoot the UV photometry (Figure 3). To ensure this, the fitter does what it can to diminish UV but not OIR flux: add dust, leading to the offsets in Figure 8, bottom-right compared to bottom-left for the systems with even trace star formation.

As to which model is "right" for PSB, while the delayed exponential fits its rest-UV photometry better than the lognormal, the mathematics of the latter prevent it from decaying rapidly enough to mimic what in reality may be a truncation of a more leisurely falling SFH. Hence, UV fluxes may not provide the answer. Also, while [O III $] / \mathrm{H} \beta$ ratios can be constrained using the lines' covariance, the $\mathrm{H} \beta$ fluxes themselves are not robust enough to support dust-corrected nebular SFRs as a cross-check (the above SED-derived $A_{V}$-SFR degeneracy precludes, e.g., Wuyts et al. 2013's continuum-based corrections). As the models disagree on rest- $J$ fluxes, incorporating Spitzer photometry may be discriminating at a spatially unresolved level, but we can turn to another sensitive test now.

Independently, a MOSFIRE $Y$-band spectrum (PI Bradač; 27 April 2017) was obtained for PSB as a slitmask filler covering rest $\lambda=4000-4400 \AA$, a region containing three key age-sensitive spectral features: $\mathrm{H} \delta, \mathrm{H} \gamma$, and the G-band. Figure 9 shows that this spectrum's much higher-resolution features are indeed well predicted by the fit to the low resolution spectrophotometry. Quantitatively, comparing these data to the delayed exponential and lognormal SFH-inferred templates yields somewhat better agreement in these lines' equivalent widths with the lognormal (Table 3). Indeed, the observed Balmer EWs are smaller-and G-band larger-than either model would predict, suggesting an older stellar population (though $\mathrm{H} \gamma$ especially may suffer infilling from resid-

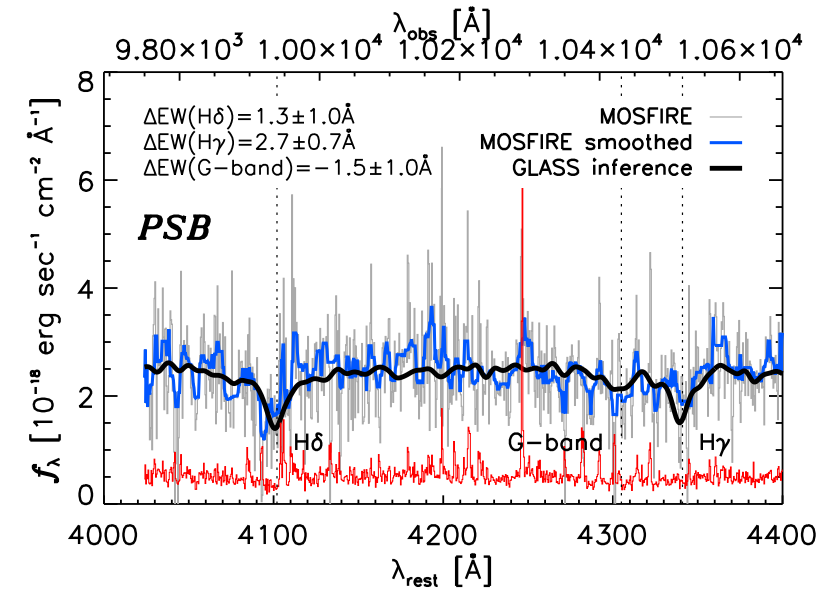

FIG. 9.- PSB's optimal lognormal model spectrum (black; broadened to $\sigma_{v}=190 \pm 40 \mathrm{~km} \mathrm{~s}^{-1}$ ) compared to MOSFIRE data (grey). RMS errors are in red; blue shows the data median-smoothed over a $10 \AA$ window. Given the implied line strengths (Table 3), the lognormal SFH reproduces these high-spectral-resolution data somewhat better than the delayed exponential, though the latter better match PSB's UV fluxes (Figure 3).

ual emission). Note: since EWs are flux ratios, absent radical asymmetries, they are insensitive to slit losses.

Notwithstanding the abrupt growth implied by the delayed exponentials (Figure 18), the above is spectroscopic evidence that such a model may not be a better description of the actual history of PSB than a lognormal, even if the latter cannot fall rapidly enough to match the system's observed rest-UV flux. For these reasons - and to further the goal of illustrating what analyses could be done with resolved SFHs covering $>$ Gyr timescales - we limit all following discussion to the spatially resolved lognormals.

\subsection{Temporally Resolved Sample Properties: Data "Longitudinalization"}

Section 4.2 resolved the galaxies in space, taking them from point- $\left(M_{*}, \mathrm{SFR}, A_{V}\right)$ to distribution-like descriptions $\left(\Sigma_{M_{*}}, \Sigma_{\mathrm{SFR}}, A_{V}\right)(r)$. This showed that systems with different sSFR $(r)$ can have similar SFR surface densities, galaxies can lie on the SFMS while their resolved components do not, and significant SFRs can persist at large- $r$ despite AGN activity.

These facts increase the burden on models aiming to describe these systems at $t_{\mathrm{obs}}$. However, the problem of validating those models' causal statements remains open. Addressing this requires empirical inferences about how these specific systems - not the galaxy population-evolved. We present a suite of such inferences here, resolving the sample in time to constrain what it most likely did and will look like.

\subsubsection{Spatially Resolved Star Formation Histories}

Figure 10 shows the sample's spatially integrated and resolved lognormal SFHs. These confirm Section 4.1's inferences: CSF has continuously formed stars at a relatively constant rate across its face, declining by only $\sim 3 \times$ since its SFH peaked at $z>4$. It is thus much more mature than its starforming counterpart SSF, which is within $\sim 1 \mathrm{Gyr}$ of its peak. A similar story holds for the passive systems: comparing peak times, PAS has been "quenching" for perhaps twice as long as PSB, though both are now farther from their peak/have SFRs that are falling faster than their starforming peers'.

As suggested by their masses, all four systems are in the declining phase of their SFHs. Yet, Figure 10 suggests this 


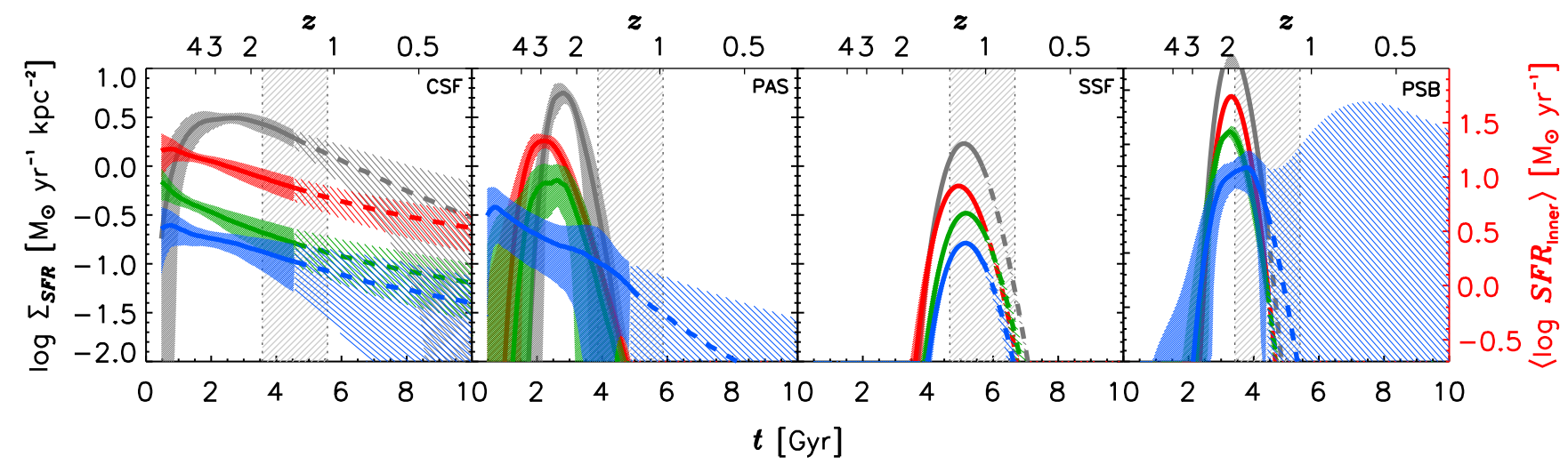

FIG. 10.- The sample's integrated and resolved SFHs. Colors correspond to previous figures with optimal extraction results (scaled to INNER surface areas) in grey. Epochs prior to $t_{\mathrm{obs}}$ are shown as solid lines/heavy shading; forecasts are dashes/light shading. Vertical bands show the $t_{\mathrm{obs}} \pm 1 \mathrm{Gyr}$ interval over which we draw longitudinal inferences (Section 4.3.2, Figure 11). Lines are medians; envelopes show 16 th-84th pctle. spreads in SFR $(t)$ from the $\left(T_{0}, \tau\right)$ PYSPECFIT covariances. All galaxies are seen after their peak, and all but CSF show signs of inside-out/bulge-first growth: INNER SFRs approach/are exceeded by MIDDLE and OUTER SFRs between $t_{\text {peak }}$ and $t_{\text {obs }}$. Conversely, the relatively bulge-free CSF (Figure 6 ) had a nearly constant SFH across its face. At least the inner $\sim r_{e}$ of PSB shut down rapidly and uniformly less than a Gyr before $t_{\text {obs }}$ (Table 2). SSF is growing strong-proportionately more so at larger- $r$ (Figures 4 , 5) — and PAS has been quenching for $\sim$ twice as long as PSB. Right $y$-axis shows absolute SFR $\mathrm{INNER}_{\text {at }}$ the sample's mean area and magnification for reference.

would not be true of their $z \sim 2$ progenitors: Except for SSFwhich grew substantially since then - these would be within a factor of $\sim 2$ of their current mass but at maximal SFR (see Figure 17). We return to this point in Section 4.3.2.

More resolved trends are shared by the three non-constant SFH systems: Compared to peak, observed SFRs in SSF, PAS, and PSB's INNER regions are closer to or below their MIDDLE and OUTER values. This is the formal signature of inside-out growth and is consistent with these systems' locations in Figure 6, where they have significantly higher $B / T$ than the constantly starforming CSF. We return to this point in Section 5, but for now note that this phenomenon has implications for the inferred progenitors of these galaxies in higher- $z$ cross-sectional datasets (Section 4.3.2).

\subsubsection{Spatially Resolved Mass Profile Histories}

With spatially resolved SFHs, we can reconstruct the mass profile evolution of the sample galaxies.

Figure 11 presents these inferences. Each row shows results for one galaxy in order of decreasing $\mathrm{SSFR}_{\mathrm{obs}}$ (SSF to PAS). From left, the panels show: 1) $\Sigma_{\mathrm{SFR}}$ vs. $\Sigma_{M_{*}}$ in each radial zone; 2) $\Sigma_{\mathrm{SFR}}$ Vs. time in each zone; 3$) \Sigma_{M_{*}}(r ; t)$ profiles at $t_{\text {obs }} \pm 1$ Gyr relative to observed INNER values (profile shape evolution); 4) $\Sigma_{M_{*}}(r ; t) / \Sigma_{M_{*}}\left(r ; t_{\text {obs }}\right)$ (total mass growth) at the same intervals (where we are willing to extrapolate the SFHs). Points in the left panels mark the epochs in the right panels.

Starting at right, all galaxies besides PAS are inferred to have gained significant $M_{*}$ at all $r$ in the Gyr preceding $t_{\mathrm{obs}}$. Further-clearest in SSF-the two starforming systems should also grow marginally in the subsequent Gyr $(\lesssim 25 \%)$.

SSF also shows explicit signs of inside-out/bulge-first growth: proportionately, OUTER regions gain more mass in this 2 Gyr period than INNER ones $(\sim 0.8$ vs. $\sim 0.6$ dex $)$. Hints of this trend appear in PSB at reduced significance.

These statements are obviously consequences of trends in the second-left panel, showing Figure 10's SFHs zoomed to the $t_{\text {obs }} \pm 1$ Gyr window. In all but CSF, INNER $\Sigma_{\mathrm{SFR}} \mathrm{S}$ fell faster than those at larger- $r$, with INNER/MIDDLE crossings close to $t_{\mathrm{obs}}$ in SSF and PSB, and over a Gyr ago in PAS.

CSF is special in this regard, however: its resolved SFHs have maintained rank-ordering over all time. Section 5.1 speculates on the potentially illuminating point of how this system escaped inside-out growth, but the left-most panel of Figure 11 bears on this question.

Replacing time with $\Sigma_{M_{*}}$ on the abscissa greatly accentuates the inside-out signature discussed above: In all but CSFbut especially PSB and SSF-INNER regions fall much faster than at least OUTER ones, moving from a high to low relative position on the $y$-axis.

Indeed, from the $M_{*}$ perspective, what is a gradual decline in SFRs with time transforms into an abrupt quenching event as galaxies appear to hit a $\Sigma_{M_{*}}$ wall at all $r$. At root, this phenomenon - the "L-shaped track" of Barro et al. (2016) is a reflection of monotonically declining sSFRs in each zone leading to asymptotic final masses. This is exacerbated by using typically logarithmic $x$-axes (as we do here), deemphasizing meaningful, if undramatic, linear increases. Indeed, the aforementioned "modest" $25 \%$ future mass growth in the starforming systems - current $\mathrm{SFR} \geq 25 \mathrm{M}_{\odot} \mathrm{yr}^{-1}$ —can correspond to $\sim 2 \times 10^{10} \mathrm{M}_{\odot}$ in CSF's case.

This fact highlights a distinction between "activity" as defined by future fractional mass growth (systems that can $>$ double $M_{*}$ in a Gyr) vs. current star formation: if the former is used, then even galaxies making tens of $\mathbf{M}_{\odot}$ of stars per year are arguably quenched, with "active" galaxies only those with sustained high sSFRs; i.e., rising SFHs.

The point spacings on the curves in Figure 11, left, clearly illustrate this: SSF's OUTER region grew by nearly $10 \times$ in $M_{*}$ over the 2 Gyr ( $\left.\sim 0.4 t_{\text {Hubble }}\right)$ probed. Contrast this with CSF's OUTER region, which grew by at most a factor of 2 despite having a higher mean SFR over this interval (Figure 17).

This leads to the question of why SFHs stop rising. Given the vast literature on this topic-_quenching"-we need not dwell on it here (Kelson et al. 2016 give an explicit mathematical framing in the above terms). However, features in these data may prove useful to that discussion (Section 5.1). For now, we note simply that CSF may provide a meaningful counterexample to the idea that $\Sigma_{M_{*}}$ is the more causally informative abscissa: the sample's most massive system, it also has the highest SFR (Table 2), the same $\Sigma_{\mathrm{SFR}}$ as SSFa system with $\sim 3 \times$ higher $\operatorname{sSFR}\left(t_{\text {obs }}\right)$ (Figure 8 ) - and has likely grown more since its SFH peaked (orange bands). As such, while it graphically emphasizes their effects, quantities besides $\Sigma_{M_{*}}$ seem key to shaping SFHs for at least CSF-like 

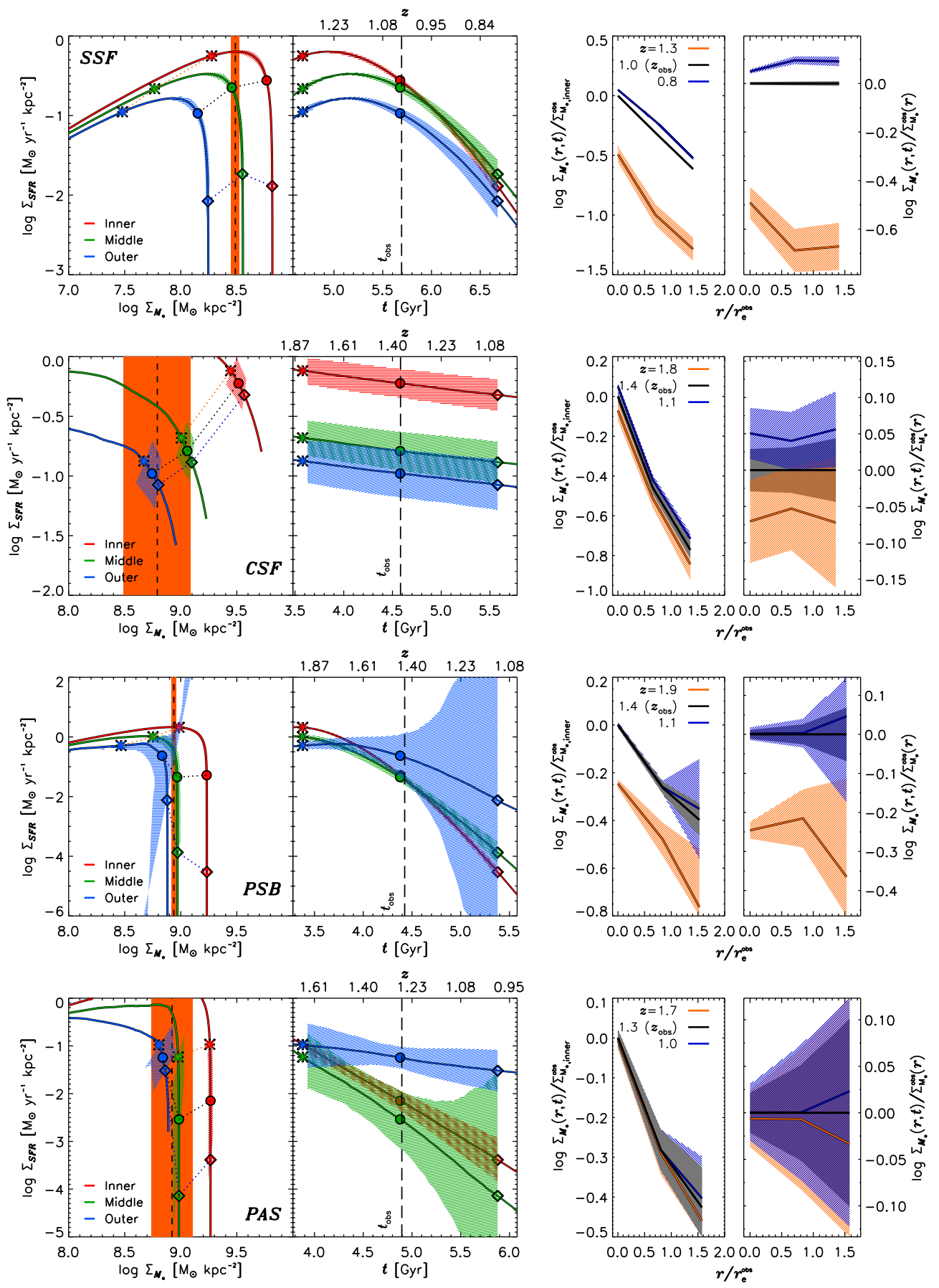

FIG. 11.- The resolved mass growth history of each of the sample galaxies (rows). From left: (1) $\Sigma_{\mathrm{SFR}}(r)$ as a function of radial $M_{*}$ surface density; (2) $\Sigma_{\mathrm{SFR}}(r)$ as a function of time since the Big Bang; (3) the $\Sigma_{M_{*}}(r)$ at $t_{\mathrm{obs}} \pm 1 \mathrm{Gyr}$ normalized to INNER values at $t_{\mathrm{obs}}$ (showing shape evolution); (4) $\Sigma_{M_{\star}}(r)$ at the same epochs normalized to the observed profile (showing total $M_{*}$ growth). Points in the left two panels denote epochs in the right two panels, and orange vertical bars show INNER $\Sigma_{M_{*}}$ at the time of peak INNER SFR. Shading shows $1 \sigma$ ranges in all cases. All galaxies but CSF show signs of bulge-first growth, visible in the resolved $\Sigma_{\mathrm{SFR}}(t)$, the change in rank-ordering of $\Sigma_{\mathrm{SFR}}\left(M_{*} ; t\right)$, or the profile evolution itself. Also with the exception of CSF (Section 5.1), the INNER $\Sigma_{M_{*}}$ at peak-SFR seems to be an asymptote for the MIDDLE and OUTER regions, even though the INNER regions themselves may grow $\sim 2 \times$ since passing this mark. This may reflect an association between bulge-building and quenching (Zolotov et al. 2015; Tacchella et al. 2016, Figure 13, below) or perhaps a coincidence given the masses/mass profiles of these systems.

(i.e., disky) galaxies (see also Lilly \& Carollo 2016).

The final inference we draw from the resolved SFHs is how these galaxies moved in the size- and $B / T$-mass planes introduced in Figure 6. We plot these projections in Figure 12.

In both panels, we show the $1 \sigma$ trajectory envelopes for each system at $z=0.5-2$. These are reconstructed by integrating the azimuthally-converted $\Sigma_{M_{*}}(t)$ at each timestep as inferred from the radially resolved SFHs (Section 4.1.2). " $B / T$ " is again taken as the mass within $r=2.5 \mathrm{kpc}$ over the total system mass at any epoch, but sizes here are half-mass radii as opposed to $r_{e}$ (though they differ little at $t_{\mathrm{obs}}$; Figure 8). Point styles show $t_{\mathrm{obs}} \pm 1$ Gyr as in Figure 11.

Three points emerge: First, despite growing by $\sim 2.5-10 \times$ in $M_{*}$, no galaxy has evolved significantly in size or $B / T$ since 


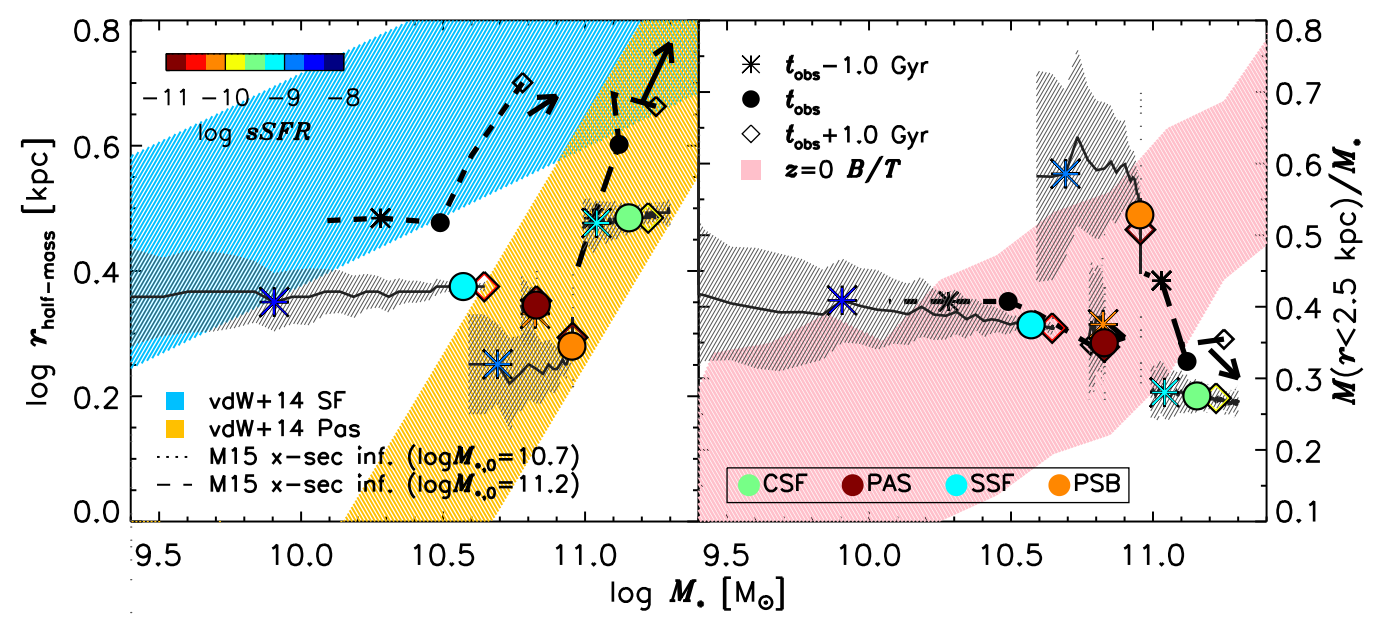

FIG. 12.- As Figure 6 but showing the longitudinally reconstructed half-mass-radius- (left) and mass-concentration/B/T-mass evolutionary trajectories of the sample galaxies. Black shading shows $1 \sigma$ envelopes across $2 \geq z \geq 0.5$, with points denoting 1 Gyr before (stars) and after (diamonds) $t_{\mathrm{obs}}$ (filled circles). Point colors show sSFR $(t)$ at the corresponding epochs. Short and long black dashes, respectively, show Morishita et al. (2015)'s cross-sectionally inferred trajectories for galaxies with the space densities of modern MW- and $\gtrsim$ M31-mass systems over the same $z$-range, highlighting the same intervals (referenced to $\langle z\rangle=1.27$ ); arrows denote their time-averaged vectors. Population loci are shaded as in Figure 6. SSF, CSF, and PSB have all grown significantly in $M_{*}$ since $z=2$, but not necessarily in size (cf. Genel et al. 2017). This behavior differs from cross-sectional inferences-even for CSF, whose mass evolution is well-captured-but $B / T$ inferences are more consistent, with galaxies tending to descend onto the fixed population locus with time from earlier states of relatively high $B / T$ (inside-out growth). At left, note that three systems will end up as small red galaxies at $z \sim 0.5$, though these will have gotten there in very different ways (Section 4.3 .2 ), highlighting the importance of path-dependence/temporal diversity underlying single-epoch parameter projections in galaxy evolution.

at least $z=2$, assuming all size evolution is due to in situ SF. Second, these (flat-ish) trajectories do not follow the galaxy population loci in any of those epochs, or indeed inferences based on fixed 2- or 3-D density $\left(r \propto \sqrt{M_{*}}\right.$ or $\left.\sqrt[3]{M_{*}}\right)$, or velocity dispersion $\left(r \propto M_{*}\right)$. Third, at least in this sample, and assuming in situ growth, longitudinal size trajectories diverge markedly from abundance-matched cross-sectional inferences (Morishita et al. 2015). Intriguingly though, $B / T$ tracks are more similar (at least for SSF). CSF's mass growth $(d x / d t)$ is also remarkably well predicted. Section 5.1.3 elaborates.

Our radial resolution and the fact that we must convert chordal (section-based) into radial (annulus-based) densities are important caveats in this context (Section 5.2). Yet, assuming we are not grossly in error-supported at least in that we predict these galaxies to have reasonable $\left(r, M_{*}, \mathrm{sSFR}\right)$ at $z \sim 0.5$ - these inferences support a few robust statements.

Regarding motion with respect to loci, echoing results in SFR- $M_{*}$ (cf. Peng et al. 2010; Speagle et al. 2014; Abramson et al. 2016), even small regions in size-mass space clearly contain galaxies that got there in very different ways. For example, at $z \sim 0.7$, three systems will appear as relatively small red galaxies (absent merging). Yet, PAS will have been there since $z \sim 2$, when PSB was a similarly small but high-sSFR "blue nugget" and SSF was an average, much lower mass starforming galaxy. Also, if anything, CSF will have traversed across the red (or parallel to but $\sim 2 \sigma$ below the blue) galaxy locus since $z \sim 2$, not along it, as suggested by cross-sectional inferences. This point emphasizes temporal diversity underlying single-epoch parameter projections in galaxy evolution.

That said, knowledge of $B / T$ and sSFR seem capable of breaking some of the above degeneracies. Consistent with the blue-nugget interpretation (Barro et al. 2013, 2016), PSB should have come from a substantially (if not abnormally) bulge-dominated $z \sim 2$ progenitor, whereas SSF's progenitor was much diskier (if still more bulge-dominated than most similar-mass galaxies). PAS's progenitor would have had a similar $B / T$ to SSF's, but significantly lower sSFR.
In all cases, if anything, $B / T$ decreases with time relative to the (quasi-static; Lang et al. 2014) population locus, if not absolutely. While another expression of inside-out growth, this signals that progenitors of modern galaxies with substantial disks $(B / T \sim 0.4)$ may descend from lower-mass galaxies with relatively high $B / T$. Interestingly-fortunately-this signal is also reflected by at least Morishita et al. (2015)'s cross-sectional, abundance-matched tracks. As discussed regarding CSF, it further suggests atypically disk-dominated galaxies as a special population. We address this point next.

\section{DISCUSSION}

To close, we interpret our results in terms of their (1) astrophysical and (2) methodological/theoretical implications. Astrophysically, we focus on the relationship between $B / T$ and SFH shape, specifically INNER sSFRs rising above global averages at the time of peak SFR, suggesting feedback from bulge building may play a role in quenching as numerical models posit. We also compare our results to predictions from previous semi-empirical models, which may similarly suggest that particularly disky galaxies - at least as defined relative to equal-mass systems - are a special class. Methodologically, we discuss this analysis' limitations, and what future studies can (and cannot) do to overcome them.

\subsection{Astrophysical Implications$$
\text { 5.1.1. Effects at } t_{\mathrm{obs}}
$$

Figures 5, 7, and 8 touch on basic astrophysical issues. These show that spatially resolved properties at $t_{\mathrm{obs}}$ can lead to different physical inferences than integrated measurements. Specifically, (1) galaxies and their components can be classified differently with respect to population loci (e.g., the SFMS); (2) systems with distinct integrated or resolved sSFR can have similar $\Sigma_{\mathrm{SFR}}$; and (3) even at $\log M_{*} \sim 11.2, \mathrm{AGN}$ do not rapidly quench SF at large- $r$ (see also Aird et al. 2012; Harrison 2014; Poggianti et al. 2017). Regarding (1), if SFHs 


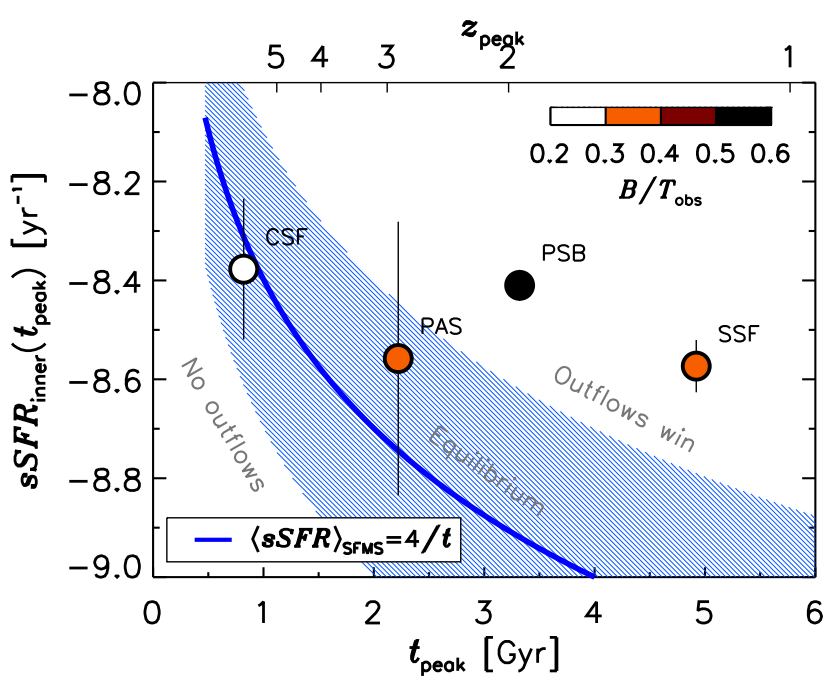

FIG. 13. - Higher $B / T$ sample galaxies (point colors) tend to have INNER SFHs that peaked above the SFMS. Meanwhile, CSF has the lowest $B / T$ and never rose above it, given the locus' elevated position at that earlier time. If the SFMS reflects an inflow/outflow equilibrium (e.g., Davé et al. 2011), this suggests a link between bulge building/strong central SF and a feedbackrelated mechanism that turns SFHs over (outflows beat inflows; e.g., Zolotov et al. 2015; Tacchella et al. 2016). If so, disk galaxies may be the objects with the flattest/most constant SFHs (Figures 10, 15; Sections 4.3.2, 5.1.2).

are lognormal, our two starforming systems go from (somewhat) underperforming pieces to normal wholes at their respective $M_{*}$. As all of these entities are evolving throughnot along-the SFMS at $t_{\mathrm{obs}}$, this fact furthers doubts about the profoundness of this locus.

\subsubsection{The Epoch of Peak INNER SFR}

Higher-level issues emerge from interrogating Figure 11. In the left-most panels, $\Sigma_{M_{*}}$ at the time of peak INNER SFR (i.e., SFH turnover) is overplotted as vertical orange bands. These span about a factor of 3 across the four systems- $-\log \Sigma_{M_{*}} \sim$ 8.5-9. In all but CSF (see below), while the INNER region itself goes on to roughly double its mass, this appears to serve as a sort-of boundary beyond which the rest of the galaxy will not pass. This may be a coincidence arising from the small sample size or these specific mass profiles (third panel), but it may also support recently popular density-related quenching mechanisms, where strong outflows at peak INNER SFRs signal the onset of galaxy-wide quenching. We find evidence for this scenario-which we interpret as a link between high- $B / T$ and falling SFHs - but also additional quenching processes.

Bearing the caveats that come with this sample size, Figure 13 presents our test. It shows each galaxy's INNER sSFRi.e., SFR per unit of mass/outflow restoring force-at the time of peak INNER SFR plotted against the contemporaneous $\langle$ SSFR $\rangle$ of starforming galaxies (Madau \& Dickinson 2014; Kelson 2014) with points coded by $B / T_{\text {obs }}$.

Hints indeed emerge that systems with high $B / T$ especially PSB and SSF-had elevated sSFR compared to the mean starforming galaxy at the epoch just before their SFHs started falling (i.e., quenching). Insofar as the SFMS reflects an equilibrium-where inflows balance outflows (Dutton et al. 2010; Davé et al. 2011) — this suggests that INNER $\mathrm{SF}$ in these galaxies was strong enough to allow feedback via, e.g., supernovae winds to quench their INNER regions, with the rest of the galaxy to follow. This would support the scenarios presented in Zolotov et al. (2015) and Tacchella et al. (2016), though here the enhanced sSFR represents a single, unique, super-SFMS peak, not the last in a series of pre-quenching surges. As mentioned (Section 4.3.2), this is also consistent with at least PSB's inferred motion in the $r_{e}-$ $M_{*}-B / T$ plane, where it went from being a small, high-sSFR, "overly" bulge-dominated blue nugget (Barro et al. 2013) to a galaxy with more normal sSFR for its $B / T$ and size.

CSF also partially supports this scenario by being a good exception to it. The centrally densest galaxy in the sample (Figure 8, top-left), it also has the highest SFR, the lowest $B / T(\sim 0.3)$, and the flattest/highest- $\tau$ SFH (Figure 10). While seemingly at odds with the above paradigm, these properties are in fact linked to it through time. Figure 13 shows that CSF peaked early enough that, though it had a high absolute $\operatorname{SFR}$ - essentially the same $\operatorname{sSFR}\left(t_{\text {peak }}\right)$ as the other systems - given the prevailing inflow conditions at the time, it need never have left equilibrium. Hence, CSF's inner region need never have blown itself out (unlike PSB and SSF's), and it could proceed to grow calmly at all $r$ through to $t_{\mathrm{obs}}$ some 4 Gyr ( $\sim 4 t_{\text {Hubble }}$ ) later, if not today (Figure 14).

This helps explain why CSF's $\operatorname{sSFR}(r)$ can be $\sim 1 / 3$ of SSF's even though they have similar $\Sigma_{\text {SFR }}(r)$ (Figure 8): it can sustain the "normal" star formation implied by the latter because its higher mass was built slowly over Gyr from a gentle SFH, not rapidly via a violent one leading to equilibrium-breaking outflows. This scenario-that both the time and timescale of SF are critical-is precisely the mathematical premise of Gladders et al. (2013, hereafter G13), and the above is qualitatively consistent with previous arguments linking bulge growth to its SFHs' shapes (see Abramson et al. 2016, Section 3.3; Section 5.1.3 below). If correct, they highlight the centrality of path-dependence in galaxy evolution, raising the need for future analyses such as this (Section 5.2).

This said, if the above is true, some additional densityindependent but time-dependent process will be necessary to shut CSF down (whenever that happens; see, e.g., Larson et al. 1980; Croton et al. 2006; Peng et al. 2015; Voit et al. 2015; Oemler et al. 2016).

How PAS fits into this story is unclear given uncertainties and the SFMS' width. While it seems more likely to have been in the "overly productive" regime of the later-peaking SSF and PSB, a robust conclusion-as well as more generally representative empirical inferences-requires additional data.

The last of Figure 11's points to emphasize is that, irrespective of their sSFR at any $t$, to the extent that lognormal SFHs reflect reality, the mode galaxy grows by $\sim 2.5 \times$ after peak SFR. While it may take gigayears to attain that asymptoteover which it will display substantial linear SFRs-this corresponds to just 0.4 dex on a $\log M_{*}$ abscissa. As such, for any SFH with a declining tail, the quenching process will almost always appear rapid. Corollary to the SFMS' case, this phenomenon would question how informative the bimodal quenched/starforming dichotomy is: Even robustly starforming galaxies — those at peak SFR! — might reasonably be "quenched" from the perspective of future $M_{*}$ growth. While perhaps useful for modeling, e.g., the evolution of mass functions, it is difficult to see how such a division illuminates the processes controlling star formation.

Regardless, in any framework, the above suggests that the best vehicles to learn about the latter are systems with rising SFHs, which are by definition low-mass. SSF's progenitors$\log \left(r_{e}, M_{*}, \mathrm{SFR}\right) \sim(0.4,9,1)$ with high $B / T$ - seem $\operatorname{good} z \sim$ 2 follow-up candidates by which to infer the development of some of today's MW-mass galaxies (Figures 5, 8, 12, 14). 


\subsubsection{Relationship to Previous Model Predictions}

In the size-mass context, Figure 12 suggests the obvious comparison between these galaxies' longitudinally inferred trajectories and, e.g., Morishita et al. (2015, hereafter M15)'s abundance-matched cross-sectional inferences for the mean system of similar-mass. Clearly, these methods yield different size projections: SSF and CSF have identical masses to M15's means at $t_{\mathrm{obs}}$, but they are both observed to be and inferred to remain smaller than what that study would predict. This is despite the fact that M15 captures CSF's size at $t_{\mathrm{obs}}-1 \mathrm{Gyr}$.

Certainly, part of the discrepancy could simply be that our sample is not average. Further, M15's cross-sectional method captures ex situ growth from mergers-which we cannotand our rather coarse radial resolution limits size-growth sensitivity. However, if substantiated by larger analyses, such tension would point to a meaningful divergence between the evolution of the mean size of galaxies and the size-evolution of the typical (i.e., mode) galaxy. This said, $B / T$ inferences seem more robust to the above issues, agreeing better qualitatively - and quantitatively, at least at $t_{\mathrm{obs}}$ - than sizes. We do not know why this is true, but it is intriguing.

If borne-out in the future, Figure 12, left's $r-M_{*}$ reconstructions would also disfavor a suite of analytic prescriptions (Bezanson et al. 2009; van Dokkum et al. 2015; Abramson \& Morishita 2016; Lilly \& Carollo 2016) wherein galaxies grow at roughly fixed $\sigma_{v}, \rho_{M_{*}}, \Sigma_{M_{*}}\left(r<r_{e}\right)$, or $\propto M_{*}^{1 / 3}(1+z)^{-1}$, corresponding respectively to tracks with slope $1,1 / 3,1 / 2$, and $\sim 0.7$ in that plane. These are far steeper than our inferences. Conversely, our tracks are not too dissimilar from the Illustris simulation's flatter $r\left(M_{*}\right)$ trajectories (Genel et al. 2017, see their Figure 5). In all cases, future longitudinal comparisons should have great potential here (Section 5.2).

Beyond such semi-empirical comparisons, we can explore how our inferences compare to the G13 global lognormal SFH model itself. This was the source of our SFH parameterization, and Abramson et al. (2016) specify ways to falsify it using these kinds of data (see their Appendix A).

The first test states that G13 would be ruled out if:

Large, abrupt discontinuities are required to explain a significant fraction of SFHs for systems dominating the universe's mass and star formation budget over a significant fraction of cosmic time (smooth, continuous parameterizations cannot describe most SFHs).

Now, of the four galaxies in this sample, one-the poststarburst PSB - has SEDs that significantly disfavor lognormal SFHs in the UV at all $r$ due to what was likely an abrupt SFH discontinuity $<1$ Gyr prior to $t_{\mathrm{obs}}$. One could thus posit that $25 \%$ of galaxies - perhaps $50 \%$ of passive ones - at $z \gtrsim 1$ and $\log M_{*}>10.5$ may therefore indeed require such events. If more data supports this conclusion, it would seriously challenge G13's limited view of the role of SFH discontinuities. ${ }^{15}$

On the other hand, PSB's MOSFIRE-derived Balmer lines are, if anything, weaker than the best-fit lognormal implies, suggesting any discontinuity occurred farther from peak-SFR than we conclude from the HST data alone (Figure 9; Table 3 ). This would make the natural lognormal fall-off respon-

\footnotetext{
${ }^{15}$ Strong Balmer absorption is common in red galaxies at these and higher $z$ (e.g., van de Sande et al. 2013; Whitaker et al. 2013; Newman et al. 2014; Belli et al. 2017), but it remains to be shown that these cannot be produced by smoothly rising and falling histories at these relatively early epochs.
}

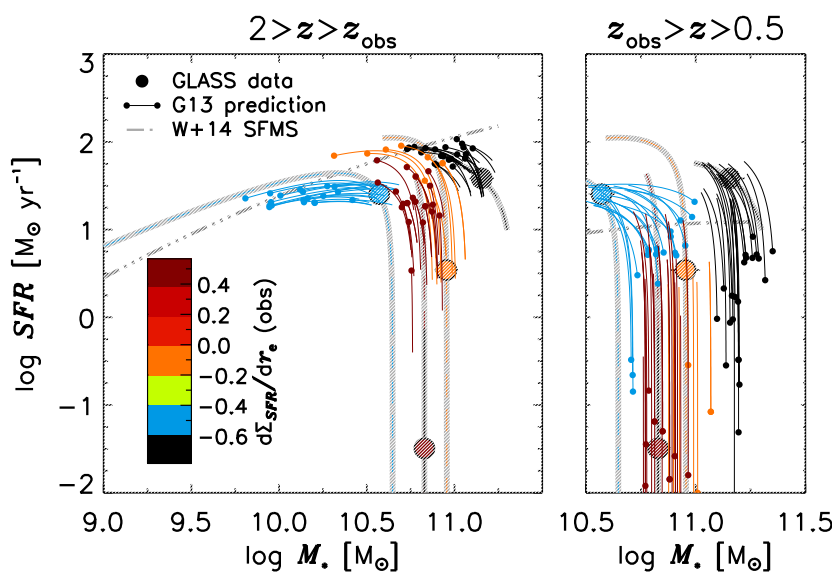

FIG. 14. - G13 model SFHs (colored lines/small points) passing through the sample at $t_{\mathrm{obs}}$ (large points) projected to show progenitor/descendant envelopes at $z=2$ and 0.5 (left/right, respectively). Colors show $\Sigma_{\mathrm{SFR}}\left(r ; t_{\mathrm{obs}}\right)$ data slopes (Figure 8; bluer $\mapsto$ more negative). G13 generally captures the data-inferred SFHs, though SSF evolves faster than predicted. Both agree that all systems were SFMS galaxies at $z=2$ (grey dot-dashes; Whitaker et al. 2014) except possibly PAS, which may already have shut down. At $z \sim 0.5$, CSF has a $\sim 50 \%$ chance of remaining a massive SF galaxy as data reconstructions suggest, while $\sim 20 \%$ of SSF's tracks suggest it will quench by then. More negative SFR gradients may imply slower evolution-another manifestation of bulge-first growth-but more data are needed to confirm.

sible for a larger portion of PSB's decline. Also, by assuming solar metallicity, we may have underestimated PSB's true metal content and the attendant UV line blanketing (Gallazzi et al. 2014). Third, we may simply have caught an unrepresentative system in our sample. Finally, two additional tests suggest that, if it is wrong about the relevance of discontinuities, G13 still captures key aspects of these data's story.

Figure 14 compares the sample SFHs to G13 model tracks passing within $2 \sigma$ of the galaxies' observed $\left(M_{*}, \mathrm{SFR}\right)\left(t_{\mathrm{obs}}\right)$ coordinate/limit. The left panel projects the models back to $z=2$, the right forward to $z=0.5$. Color coding reflects the slope of the data's observed SFR surface density profiles.

In this independent cross-check, G13's high-z predictions match the data inferences to within about a factor of 2 in both axes in all but SSF, which has grown much faster than the model tracks. Given that G13 was based on no SED fits and contained no data-derived SFH information whatsoever, this level of agreement is encouraging.

Looking ahead to $z=0.5$, the model also does reasonably well, if perhaps in a more probabilistic sense. Around $20 \%$ of model SFHs passing near SSF at $t_{\text {obs }}$ suggest it will become a passive, $\log M_{*} \sim 10.7$ low- $z$ galaxy; $\sim 1.3 \sigma$ agreement with its (median) data-inferred trajectory. Similarly, about half of CSF-matching trajectories suggest it will remain a massive, starforming system. Clearly, all inferences agree PSB and PAS will become $\sim \mathrm{M} 31$ - and MW-mass passive systems.

Note: these statements are converse to those in Section 4.1.2: Figure 5 shows where $z=0$-selected MW-mass progenitors lie at mean sample redshift; Figure 14 shows where the descendants of $z>1$ selected galaxies lie closer to today.

In terms of informing future G13-like modeling, the spatially resolved data suggest a $d \mathrm{sSFR} / d t-d \Sigma_{\mathrm{SFR}} / d r\left(t_{\mathrm{obs}}\right)$ anticorrelation, such that galaxies with the least-negative SFR gradients are evolving the fastest (yet another restatement of inside-out growth). However, SSF's data buck this trend, so robust statements await larger samples.

Figure 15 presents a final test. Here, we plot the data against 


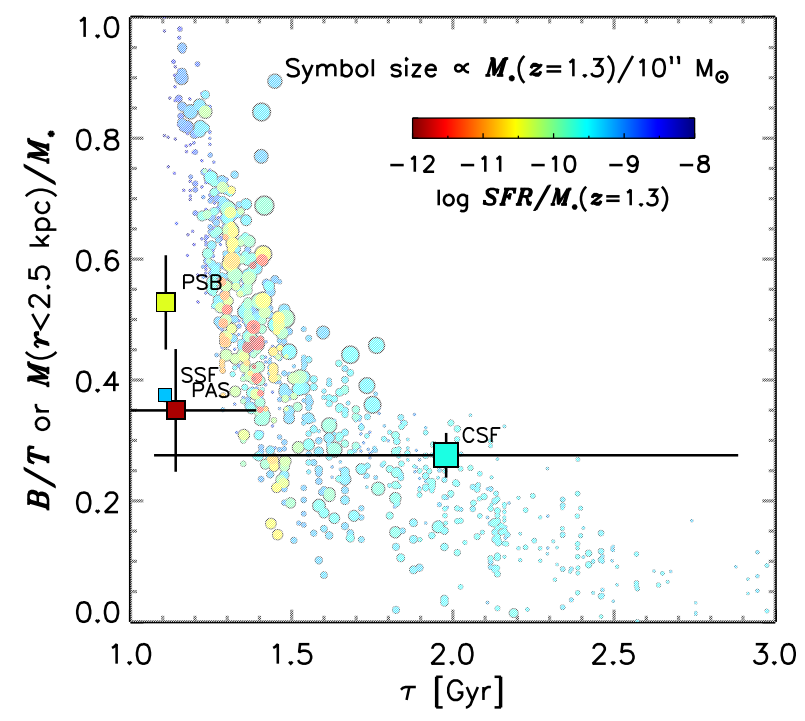

FIG. 15.- GLASS sample data inferences (squares) vs. G13 $B / T-\tau$ predictions (circles; see main text/Abramson et al. 2016, Figure 14 for details). Colors show model $z=1.27=\left\langle z_{\text {GLASS }}\right\rangle$ and observed sSFRs. The data's trend for higher measured $B / T$ to correspond to smaller inferred $\tau$ and the corresponding sSFRs is well predicted, though and SSF and PSB are quantitatively low. This amplifies Section 5.1.2's discussion of a bulge-growth/SFH shape connection-high (low) $B / T$ implies SFHs that do (not) turn over-a robust conclusion of this work and an independent consequence of G13. Indeed, if CSF's $\tau$ error bar-and perhaps G13's large- $\tau$ tail-reflect the fact that they cannot formally be captured by a lognormal, this may suggest that galaxies with near-constant SFHs form a special class.

the $B / T-\tau$ prediction from Abramson et al. (2016, see their Figure 14). This is calibrated at $z=0$ by rank-ordering G13 $\tau$ and Gadotti (2009) $B / T$ measurements at fixed $M_{*}$ assuming the smallest $\tau$ corresponds to the largest $B / T$. Point colors reflect $z=1.27=\left\langle z_{\text {GLASS }}\right\rangle$ model and observed sSFRs. Since no information on the $y$-axis was used in its construction, the properties of this locus represent blind predictions of the G13 model. As such, this comparison is a true experiment.

Two points are salient. First, the general $B / T-\tau$ trends in the data reflect those in the G13 model: smaller inferred $\tau$ is associated with systems of higher measured $B / T$. Second, the sample's measured sSFRs (point colors) are consistent with those of the G13 tracks at the appropriate $B / T$, though they are likely more massive than the typical model at that $y$ location, and SSF and PSB have quantitatively lower $\tau$ than predicted. CSF - the lowest- $B / T$ sample galaxy-is especially well-matched in this regard. Considering the model locus was calibrated at $z=0$ - some eight Gyr after the epoch probed here-we consider even this level of agreement somewhat striking. [Given their declining SFHs, the GLASS sample's $B / T$ should evolve little between $t_{\mathrm{obs}}$ and today (Figure 11).]

If supported by larger future datasets, the implications of especially CSF's location in Figure 15 amplify Section 5.1.2's discussion of the connection between $B / T$ and SFH shape as captured by $\tau$, the SF timescale: Apparently, G13 prefers galaxies with $\tau \gtrsim 1.5 \mathrm{Gyr}$ - i.e., the flattest SFHs - to be the most disk-dominated, even if they are relatively massive. ${ }^{16}$

\footnotetext{
${ }^{16}$ Note that constant SFHs cannot formally be captured in the lognormal framework. It is thus interesting to consider whether the large- $\tau$ tail in the G13 locus in Figure 15-or even CSF's $\tau$ error bar-fundamentally highlights a physical regime in which this paradigm breaks down (or something
}

This link between high (low) $B / T$ and SFHs that do (not) turn over is perhaps the central physical inference of this work, and also an independent feature of G13.

\subsection{Implications for Methods and Theory 5.2.1. Future Applications}

While other studies have used similar spectrophotometric data to reconstruct aspects of galaxies' longitudinal evolution at $z>1$ (Whitaker et al. 2013; Newman et al. 2014; Nelson et al. 2016b; Domínguez Sánchez et al. 2016; Lee-Brown et al. 2017; Toft et al. 2017), $S / N$ and spatial resolution limitations have forced these to rely on stacks, treat galaxies as monolithic objects, or quote SFH summary statistics (e.g., age). By exploiting gravitational lensing and GLASS' depth, this is, as far as we are aware, the first $z>1$ study to perform a full, spatio-temporally resolved, spectral continuum SFH reconstruction for individual galaxies to $r \sim 2 r_{e}$.

To progress, more such (or better) information will be essential: Figures 8, 10, 11, 12 are (hopefully) crude representations of the kind of empirical inferences that will ultimately be available to constrain the galaxy evolution narrative.

Indeed, next generation facilities will likely allow this type of analysis to become routine. With its redder, higher spectral and spatial resolution slitless grisms, slitted spectrographs, IFU, and imagers, JWST could apply more sophisticated versions-incorporating new insights into spatially variable emission line interpretations (Sanders et al. 2017) - to practically any suitably bright $z \gtrsim 0.7$ system. Further, it will extend samples vastly in redshift and provide critical spatially resolved $M_{*}$ constraints from rest-IR photometry that do not yet exist (and whose absence is a key weaknesses of this study).

WFIRST will more directly translate our methodology, but over huge areas of the sky, enabling tests of the critical galaxy-environment connection (perhaps the key issue Dressler 1980; Kelson et al. 2016). Combined with $30 \mathrm{~m}$ class telescope observations of specific targets-which will replicate some of JWST's capabilities but at higher spatial resolution, given planned adaptive optics systems - this may prove revolutionary. Lastly, as envisioned, spectrographs on all of the above platforms will measure spatially resolved stellar absorption lines, providing more robust SFH and metallicity constraints (e.g., Figure 9; Worthey et al. 1992; Trager et al. 1998), another datum we could not incorporate here. To our knowledge, Newman et al. (2015) and Toft et al. (2017) present the only such observations at $z>1$ for two $\log M_{*} \sim$ 11.1-11.5 lensed systems, with LEGA-C (van der Wel et al. 2016) just now providing the first statistical database at $z \sim$ 0.7. Next generation facilities may make such data the norm.

Other meaningful immediate advances include: moving to true radial reconstructions using IFUs, building a chordal-toradial transformation calibration library using, e.g., GALFIT (Peng et al. 2002), and exploring the effects of moving from parametrized to free-form SFHs. The latter were adopted by the Carnegie Spitzer IMACS Survey (Kelson et al. 2014; Dressler et al. 2016; Dressler et al., in preparation), and this and similar approaches based on halo merger-tree motivated SFHs (Pacifici et al. 2013, 2016) and fine landscapes of basis functions (Iyer \& Gawiser 2017) are proving powerful. Specifically, these have the advantage of avoiding the assumption that SFHs rise and fall/each phase's relative mass contribution, and allowing each SFH component to have its own

more abstract). If so, SFHs approaching this limit may form a special class. We encourage further investigation in this vein. 
$A_{V}$ and metallicity, enabling self-consistent chemical enrichment and mass-growth modeling (Muñoz \& Peeples 2015). Both have potentially important ramifications we could not assess, and, though SFH discontinuities may complicate temporal, e.g., size reconstructions, further study will be fruitful.

\subsubsection{Irriducible Uncertainties}

Despite the above advances, a core issue will accompany all future work. Assuming the above techniques yield consensus SFHs when applied to data of suitable quality, and irrespective of whether those data are resolved radially, in sections, or treated as independent spaxels, the results will never correspond to the evolution of the matter in that aperture. Rather, they will reflect the evolution of the aperture based on its contents at $t_{\mathrm{obs}}$. Because we only ever get one look at a galaxy, regardless of the fineness of the empirical mesh, inferences derived therefrom must follow from each cell's state at one and only one instant. Yet, the galaxies themselves are collections of moving elements, so the stars/gas we aim to characterize may never occupy that cell at any other epoch. This fact-which also forces assumptions about the role of mergers, which contribute to mass growth without leaving unique spectral signatures-has been an ever-present if inevitable issue here, whose effects only forward modeling can constrain.

Of course, it may be that, once $t_{\text {Dyn }} \ll t_{\text {Hubble }}$ (which occurs quite early), material is moving so swiftly compared to the timescales of interest that the above effects "average-out," becoming a non-issue. Or, perhaps due to secular evolution, they may not. Either way, observers and simulators should work together to understand and constrain the effects of this fundamental empirical limitation, thus clarifying when to conclude the project of understanding how galaxies' individual biographies led them to their observed states.

\section{SUMMARY}

Based on deep, 17-band CLASH/HFF + G102/141 GLASS HST spectrophotometry, we reconstruct the integrated and spatially resolved star formation histories of four, $z \sim 1.3$ cluster-lensed galaxies (Table 1) using full-spectrum, restUV/-optical fitting at $|r| \lesssim 2 r_{e}$. The sample spans $\log M_{*} \simeq$ 10.5-11.2 and contains one anciently passive, one recently quenched, one continuously starforming, and one strongly starforming galaxy (Table 2; Section 4.1), enabling these case studies to probe spatio-temporal phenomena relevant to meaningful parts of parameter space (MW- to super-M31-mass progenitors; Figure 5). Our work has three main implications:

Methodologically, the best current data enable observers to resolve galaxies in both space and time, offering new ways to empirically connect their observed properties-here, $r_{e}, B / T$, $\Sigma_{M_{*}}(r), \Sigma_{\mathrm{SFR}}(r)$ (Section 4.2, Figure 8); perhaps one day $A_{V}(r), Z(r)$ - to aspects of their individual histories, moving data-driven inferences much closer to the domain of theoretical models. We demonstrate this using just three radial bins and the assumption of lognormal SFHs (Sections 2,3), but future IFU studies or different SED fitting techniques could relax these constraints, enabling robust assessments of the dependence of astrophysical conclusions on such factors.

Astrophysically, we find hints of a connection between $B / T$ and SFH shape/spatial uniformity (Sections $4.3,5.1$ ). The three sample galaxies with the highest $B / T$ are also those showing signs of inside-out growth-where the INNER regions move from leading to lagging those at larger- $r$ in $\operatorname{SFR}(t)$ - and indeed those with obviously rising and falling
SFHs (Figures 6, 10, 11). Further, two of these are inferred to have had sSFRs well above the SFMS at the time of peak INNER SFR (with the other marginally elevated). This is to be contrasted with the diskiest system $(B / T \sim 0.3)$, which had a spatially uniform, slowly declining SFH that never exceeded the mean sSFR of all starforming galaxies (Figure 13). Both signals are consistent with recent theoretical suggestions positing a link between bulge growth and SFR declines (Zolotov et al. 2015; Tacchella et al. 2016).

Further, individual size- and $B / T$ evolutionary trajectories may diverge from cross-sectional inferences based on abundance matching (Morishita et al. 2015, Figure 12), emphasizing the temporal diversity underlying single-epoch galaxy parameter projections. Half-mass radii are inferred to evolve negligibly across at least the $2 \mathrm{Gyr}$ bracketing $t_{\mathrm{obs}}$, even as galaxies gain factors of $\sim 6$ in $M_{*}$ over the same period. Similarly, $B / T$ evolution may be flat or decreasing in an absolute sense - suggestive of inside-out growth - but is more robustly negative relative to the mean at a given $M_{*}$; i.e., sample galaxies tend to start above the $B / T-M_{*}$ locus at low mass, but endup at the appropriate value at their terminal masses, in closer agreement to M15's cross-sectional inferences.

While sample size forces these statements to remain tentative, JWST, WFIRST, and the $30 \mathrm{~m}$ class telescopes will routinely produce data that can support this and more sophisticated analyses, and therefore contextualize or refute them.

Finally, information-theoretically, empirical spatiotemporal inferences will ultimately be limited in that they must forever be based on the matter in an aperture at one and only one epoch. As such, even given arbitrarily well resolved, high-S/N IFU data and no systematic uncertainties (e.g., as to the IMF or chemical evolution), the fundamentally Eulerian nature of data-driven reconstructions will always place them in a slightly different domain (aperture evolution) than the Lagrangian theories they should be used to test (galaxy evolution). Understanding the relationship between these two entities will require the joint efforts of theorists and observers working towards a common interpretive framework. We are hopeful such partnerships will soon emerge, ushering in a qualitatively new relationship between data and theory.

LEA thanks Dan Kelson, Jordan Mirocha, and Simon Birrer for their toleration during many enlightening conversations. The same goes for Lindsay Young, who illuminated key longitudinal/cross-sectional distinctions underpinning this work, and Mike Gladders, whose ideas were central to it. BV acknowledges support from an Australian Research Council Discovery Early Career Researcher Award (PD0028506). GLASS (HST GO-13459) is supported by NASA through a grant from STScI operated by AURA under contract NAS 5-26555. This work uses gravitational lensing models by PIs Bradač, Natarajan \& Kneib (CATS), Merten $\&$ Zitrin, Sharon, Williams, Keeton, Bernstein \& Diego, and the GLAFIC group. Lens modeling was partially funded by STScI's HST Frontier Fields program; models were obtained from the Mikulski Archive for Space Telescopes (MAST).

\section{Facilities: HST ACS/WFC3; Keck MOSFIRE.}

Software: IDL Coyote (D. Fanning; http://www.idlcoyote.com) and MPFIT (Markwardt 2009) packages; SAO DS9. 


\section{APPENDIX}

\section{A: DATA STRUCTURES}

Spectra - The GLASS pipeline outputs a FITS cube for each source in each grism at each orientation. The files contain the following objects/data types:

- DSCI - Direct image of each source taken from a CLASH or HFF WFC3IR image stack to which the GLASS full field grism frames are aligned/referenced. $(60 \times 60$ pix image; "DIRECT_IMAGE" in text.)

- DWHT - DSCI's RMS error map. (60×60 pix image.)

- SCI - Cutout of the full field GLASS grism image containing a target's 2D spectrum. ( $360 \times 60$ pix image.)

- MODEL - Model of the target's spectrum in SCI; based on the CLASH/HFF alignment image SExtractor segmentation map. $(360 \times 60$ pix image. $)$

- CONTAM - Model of all non-target spectra in SCI; based on the CLASH/HFF alignment image SExtractor segmentation map. $(360 \times 60$ pix image. $)$

- WHT - SCI's RMS error map. (360×60 pix image.)

- YTRACE - Centerline of the target's 2D spectrum (spectra are not rectified). (360 element array.)

- SENS - 1D grism response curve along YTRACE $\left(e^{-} / \mathrm{s} \rightarrow 10^{-17} \mathrm{erg} / \mathrm{s} / \mathrm{cm}^{2} / \AA\right)$. (360 element array.)

- WAVE $-\lambda(x)[\AA]$ along YTRACE. (360 element array.)

All 2D data derive from interlaced-not drizzledWFC3IR reductions, maintaining pixel-to-pixel independence. 1D spectra derive from the SCI minus CONTAM image, masking SCI $=0$ pix, and those with CONTAM $\geq 2 \times$ WHT $\& \&$ CONTAM/MODEL $\geq 0.01$. (I.e., pixels with significant contamination bright enough compared to the target to cause meaningful continuum biases over $\sim$ hundred pixel extents.) Quality control (Section 2.2) ensures masking is minimal for the sources in this study, but it is visible, e.g., in the OUTER zone of CSF (Figure 3).

Spectral extraction boxes are centered on and reflected over YTRACE $(x)$ with widths set by $r_{e}$ as measured from DSCI (Section 2.3). Spectral uncertainties at each $\lambda$ are the quadrature sum of $\operatorname{WHT}(x)$ over all unmasked $y$ in the extraction zone.

For the optimal extractions, each column of a SCI-sized image is filled with an object's normalized 1D spatial profile centered on YTRACE $(x)$. This weights the SCI - CONTAM flux sum over all $N \leq 60$ unmasked $y$ pixels at each $x$ (Horne 1986).

This process is repeated independently in G102 and G141, producing a pair of spectra and LSFs for each zone of each object. These are sent to PYSPECFIT simultaneously, along with multiband, aperture-matched CLASH+HFF photometry.

Photometry - In addition to the spectra, $N=17$ band image stacks are also cut for each source from PSF-matched (F160W resolution) CLASH/HFF imaging (Morishita et al. 2017). These and their weight maps are rebinned from their native 0 ."06 to GLASS's 0 ." 065 pixel scale and rotated to the

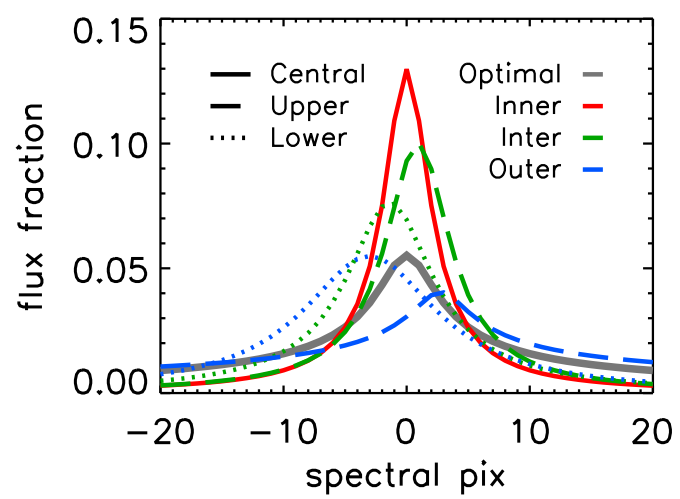

FIG. 16. - LSFs for CSF's unfolded radial and optimal extractions showing offsets and chord profile variations (Section 2.3). These preclude a priori impositions of symmetry in objects with PA $\sim 45^{\circ}$ if studying line widths.

GLASS DSCI image orientation by finding the angle $\theta / \mathrm{deg} \in$ $[0,359]$ that minimizes

$$
\chi_{\theta}^{2}=\sum_{\text {pix }} \frac{\left(I_{\theta}^{\mathrm{NIR}}-\mathrm{DSCI}\right)^{2}}{\mathrm{DWHT}^{2}},
$$

where $I_{\theta}^{\mathrm{NIR}}$ is a stack of the F105-160W CLASH/HFF stamps (rotated by $\theta$ ). The best-fit $\theta$ is applied to each CLASH/HFF image individually. This angle will generally not correspond to the GLASS orientation-the HST roll angle when the spectra were taken-as DSCI is from an independent alignment image. Pixel interpolation is cubic.

Once properly orientated, each band of CLASH/HFF imaging is photometered in the same zones as the spectral extractions. We assume YTRACE follows the NIR image centroid, which we find using SExtractor on the rotated F105-160W stamp stack, $I^{\mathrm{NIR}}$. As with $\theta$, we fix this centroid to $r=0$ in all bands (in the blue, prominent starforming regions can and do shift independent SExtractor $\mathrm{X}_{-}$and Y_IMAGE estimates). The $r_{e}$-based extraction zone offsets derived from the DSCI light profile (Section 2.3) are then applied based on this centroid and all flux in the CLASH/HFF stamp summed within each zone $\left(60 \times y_{\text {zone }}\right.$ pix aperture). Comparisons to synthetic photometry derived directly from the GLASS spectra suggest this process is absolutely accurate to within $\pm \sim 30 \%(1.5 \sigma)$. Regardless, the spectra are scaled to the photometry-which is corrected for galactic extinction-during SED fitting (Section 3). Figures 2 and 3 demonstrate that relative spectrophotometric accuracy is excellent.

\section{B: FURTHER DETAILS ON SPECTRAL SYNTHESIS MODELING \\ Fitting Scheme}

The fitting scheme is as follows:

1. Assume a delayed exponential SFH;

2. Find the galaxy's global redshift and integrated properties using the optimal extraction;

3. Find the redshift and properties of the INNER extraction using the redshift from Step 2 as strong prior;

4. Lock the MIDDLE and OUTER extractions to INNER's redshift, shifting the traces by the offsets identified dur- 
ing LSF measurement if these are larger than the formal $z$ uncertainty (Section 2.3).

5. Find the properties of the MIDDLE and OUTER extractions given the central redshift solution;

6. Repeat Steps 2-5 assuming a lognormal SFH fixed to the correct redshift.

\section{Fitting Parameters}

Details differ slightly depending on the SFH, but the full parameter and prior set is as follows:

- Redshift $(z)$ in the case of the delayed exponential model and optimal/INNER extractions. Broad gaussian prior based on GLASS database redshifts.

- Stellar mass $\left(M_{*}\right)$ and star formation rate (SFR) in all cases. No prior.

- Equivalent widths and fluxes for $\mathrm{H} \alpha, \mathrm{H} \beta$, [O III] $\lambda \lambda 4959,5007$, [S II] $\lambda \lambda 6718,6733$ in all cases. Uniform priors from $[0,500] \AA$ for $\mathrm{H} \alpha$ and [O III]; [0, 50] for [S II]. Balmer ratios fixed to unextinguished values in the delayed exponential SFH; free for lognormals.

- Dust extinction to the stellar continuum, $A_{V}$. Uniform [0,3] mag prior; Calzetti et al. (2000) reddening law.

- SFH parameters- $-\left(\right.$ age, $\left.\tau_{\exp }\right)$ or $\left(T_{0}, \tau\right)$ for delayed exponential and lognormal parameterizations, respectively. Priors are: age-log-uniform in $\left[10^{7}, 10^{10}\right] \mathrm{yr}$; $\tau_{\text {exp }}$ - $\log$-uniform in $\left[10^{7}, 10^{10}\right] \mathrm{yr} ; T_{0}$ - $\log$-uniform in $\left[10^{9}, 10^{10}\right] \mathrm{yr} ; \tau$ - uniform in $[0.1,3] \ln \mathrm{Gyr}$.

Emission lines are fitted jointly with the stellar continuum/corresponding absorption, but, beyond CSF AGN identification (Figure 7) and gross $\mathrm{EW}(\mathrm{H} \alpha+[\mathrm{N} \mathrm{II}])$ trends (Figure $19)$, we defer a discussion of these to a later paper.

Both passes use a $\chi^{2}$ likelihood function (see Appendix B of Newman et al. 2014), but we quote the medians of the resulting posterior distributions as parameter estimates, which are more robust than those from the best-fit model.

\section{C: SFH RECONSTRUCTION SELF-CONSISTENCY}

A concern may be that inferences derived from summing the INNER, MIDDLE, and OUTER results diverge from those based on the integrated optimal extractions. At $\lesssim 1.2 \sigma$, this is not the case at $t_{\mathrm{obs}}$, and fortunately over much of cosmic time.

Figure 17 presents this cross-check, showing $\operatorname{SFR}(t)$ and $M_{*}(t)$ inferred from the integrated vs. summed resolved results. These are consistent, albeit within large uncertainties on the sums for the passive PAS and PSB systems. The fact that all four galaxies are past their peak SFRs (Figure 10) is brought to the fore here, with no system likely to gain "substantial" mass in the future. This highlights a meaningful distinction between starforming and rapidly growing galaxiesthose with rising SFHs (Sections 4.3.2, 5) - but the agreement between integrated and summed inferences when SSF and PSB were rapidly growing is nevertheless reassuring.

\section{D: DELAYED EXPONENTIAL RESULTS SUMMARY}

Figure 18 shows some relevant results based on the delayed exponential SFHs not used in the main analysis. The top panel

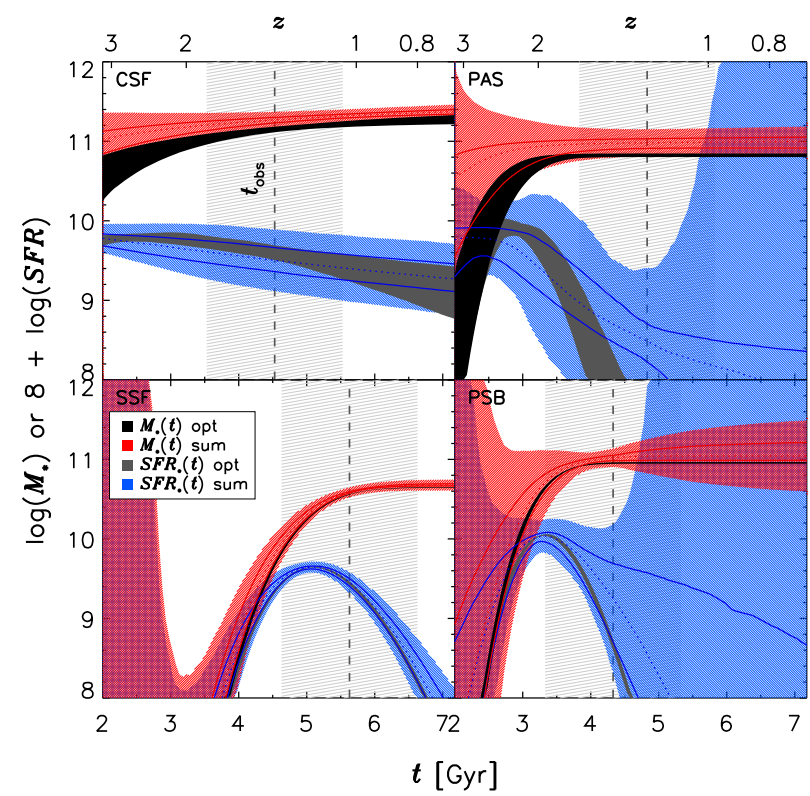

FIG. 17.- SFHs and mass growth curves from the optimal integrated extractions (black/grey, respectively) vs. those summing INNER, $2 \times$ MIDDLE, and $2 \times$ OUTER results (blue/red, respectively). Vertical dashes show $t_{\text {obs }}$ with the \pm 1 Gyr projection window shaded (Section 4.3.2). $1 \sigma$ summed uncertainties are calculated as a fraction of the median (shading), and as the log of the high/low linear $\operatorname{SFR}(t)$ or $M_{*}(t)$ estimates (solid lines). The former leads to mostly unconstrained (future) summed uncertainties for PAS and PSB due to their low $\operatorname{SFR}\left(t_{\text {obs }}\right)$. Even so, the largely independent sum of the parts and inferences from the whole broadly agree.

shows spatially resolved age inferences (left; age $\equiv t_{\text {obs }}-t_{\text {start }}$; Equation 1$)$, and $e$-foldings (right; age $\left./ \tau_{\text {exp }}\right)$. The bottom shows the resolved SFHs themselves (replicating Figure 10).

A similar picture emerges from these panels as with the lognormal SFHs, though its expressed slightly differently/less conveniently given our intent. First, from the top-left panel, inside-out age gradients characterize PAS and SSF (INNER regions are oldest). While, unlike in the main analysis, PSB does not appear to have such a gradient, the top-right panel pushes back on this, showing an inside-out $e$-folding, i.e., $m a$ turity gradient (INNER regions most evolved).

Figure 18, bottom, elaborates on this swap: PSB has $\delta$ function-like (zero-age) best-fit exponential SFHs. This follows from discussions in Sections 4.1, 4.2 regarding how this form expresses what is likely an abruptly truncated (poststarburst) SFH. The other systems have $e$-folding trends that mimic the lognormal inferences, with the starforming systems having fewer (i.e., wider SFHs) and PAS having the most.

Indeed, the starforming delayed exponential SFHs grossly correspond to the lognormals (Figure 10), especially for CSF, where the same quasi-flat, rank-ordered SFHs emerge at all $r$. SSF has similar INNER properties, but to capture this system's sSFR gradient (Figure 8), the MIDDLE and OUTER SFHs become dramatically compressed, illustrating the this form's expression of inside-out growth. (Note, however, that SSF's INNER SFH still moves from a leading to lagging position roughly at $t_{\mathrm{obs}}$ as in Figure 11.) The abruptness of these histories - and those for the the passive systems - contributes to our disfavoring them for the time-domain reconstructions that at the core of the main analysis. 

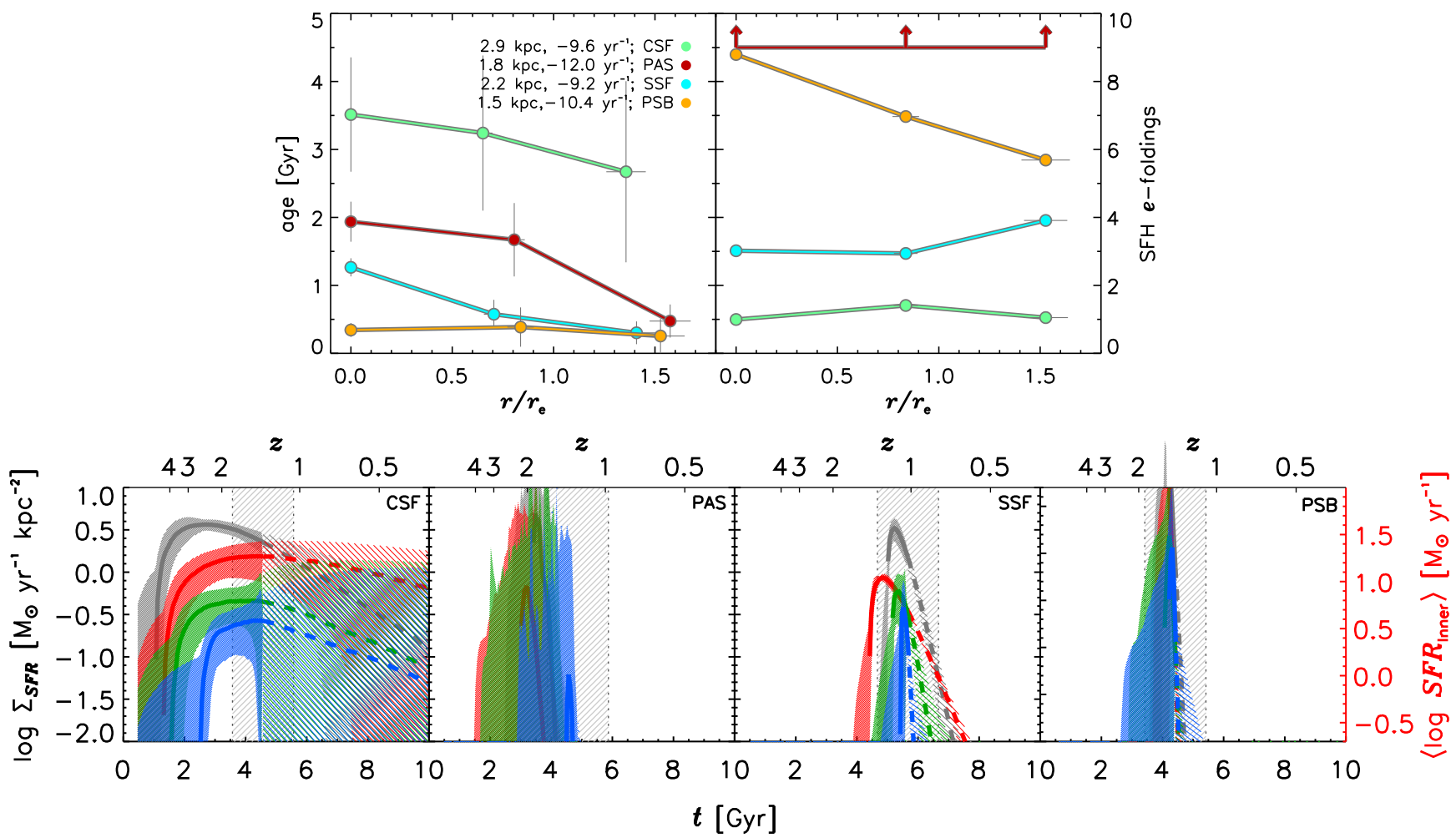

FIG. 18. - Summary plots based on the delayed exponential SFH fits (Section 3$)$. The top panels show radial parameter trends - age $\equiv t_{\mathrm{obs}}-t_{\mathrm{start}}, e-$ foldings $=$ age $/ \tau_{\text {exp }}$-which reveal similar signatures of inside out growth to the main, lognormal analysis (INNER regions older/having undergone more $e$-foldings). The bottom panels reproduce Figure 10, showing the SFHs in full. These qualitatively reflect the lognormal results for CSF and SSF's INNER region, but PSB and PAS become essentially a convolution of $\delta$-functions, implying unrealistic growth rates in PSB's case and forbidding time-domain reconstructions. Note that inferences such as the INNER/OUTER SFH crossing epoch (inside-out transition) in SSF agree using either method (cf. Figure 11).
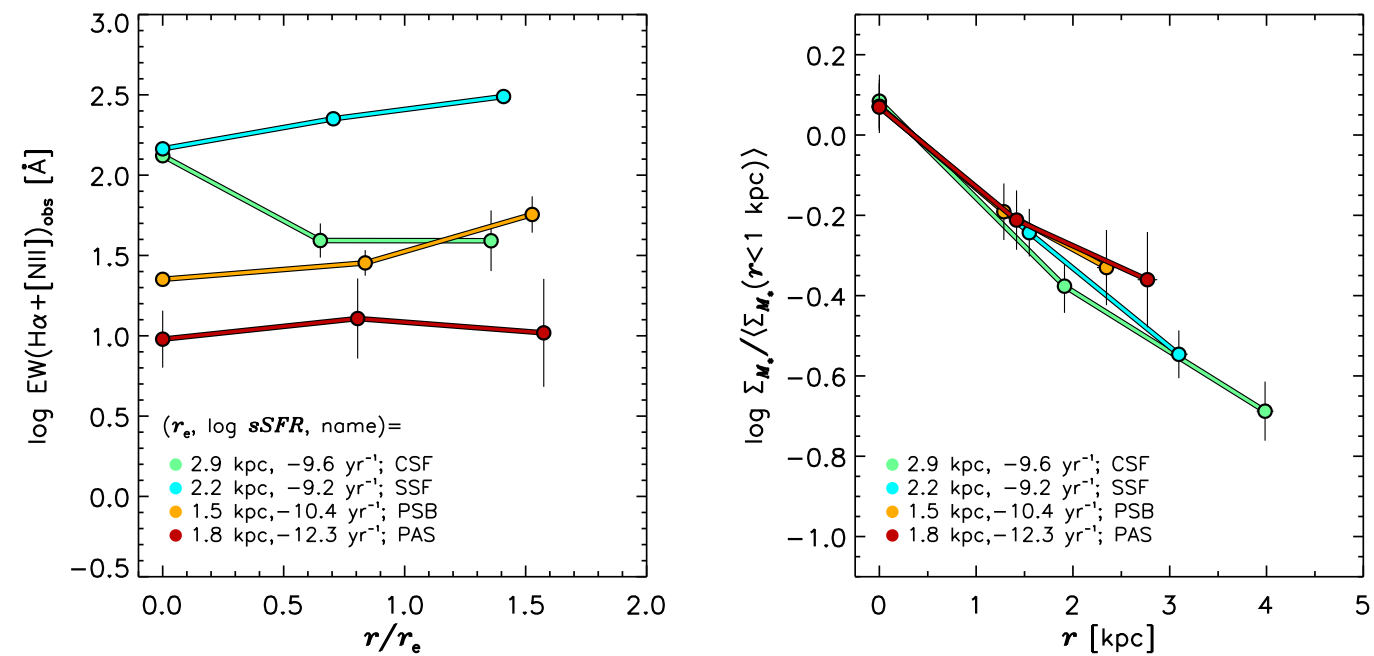

FIG. 19.- Reinterpretations of Figure 8. Left: Dust-uncorrected EW(H $\alpha+[\mathrm{N}$ II] $)$ yield similar trends to SED-fit sSFRs. CSF is an exception: AGN activity elevates the INNER inference (Figures 3,7), while those at larger- $r$ are $\sim 10 \times$ depressed relative to SSF. IFU data will reveal if this is due to increased extinction towards CSF's H II regions or a real suppression of sSFR beyond the factor of $\sim 3$ inferred from the full SED. Right: Inner kpc-normalized $\Sigma_{M_{*}}$ profiles vs. physical radii (as opposed to $r / r_{e}$ ), highlighting the sample galaxies' sizes. Trends are the same as in the main-text figure, with the larger starforming systems having steeper mass profiles than the passive ones, perhaps suggesting the latter have already some dry merging (Section 4.2; e.g., Newman et al. 2012).

\section{E: $\mathrm{EW}\left(\mathrm{H} \alpha+[\mathrm{N}\right.$ II] $)$ TRENDS, $\Sigma_{M_{*}}(r)$ IN PHYSICAL RADII, AND THE $z \gtrsim 1 \Sigma_{M_{*}}-\Sigma_{\text {SFR }}$ LAW}

Figure 19 reinterprets Figure 8's top- and middle-right panels using dust-uncorrected $\mathrm{EW}(\mathrm{H} \alpha+[\mathrm{N} \mathrm{II}])$ as an sSFR proxy (left), and physical—not $r_{e}$-normalized-radii (right). These yield very similar trends to those discussed in-text and preserve the systems' rank ordering. The exception is CSF's EW profile, where, compared to $\operatorname{SED}$-derived $\operatorname{SSFR}(r)$, IN- 
TABLE 4

DELAYED EXPONENTIAL-DERIVED INTEGRATED PROPERTIES FROM OPTIMAL EXTRACTIONS

\begin{tabular}{cccc}
\hline \hline Tag & $\log M_{*} / \mathrm{M}_{\odot}{ }^{\mathrm{a}, \mathrm{b}}$ & $\log \mathrm{SFR} / \mathrm{M}_{\odot} \mathrm{yr}^{-1 \mathrm{a}, \mathrm{b}}$ & $A_{V}[\mathrm{mag}]$ \\
\hline PAS & $10.77 \pm 0.07(0.10)$ & $<-9.13^{\mathrm{c}}$ & $0.25 \pm 0.13$ \\
SSF & $10.54 \pm 0.06(0.09)$ & $1.58 \pm 0.06(0.07)$ & $1.31 \pm 0.03$ \\
PSB & $11.05 \pm 0.07(0.10)$ & $1.16 \pm 0.15(0.27)$ & $1.50 \pm 0.09$ \\
CSF & $11.17 \pm 0.07(0.16)$ & $1.65 \pm 0.14(0.20)$ & $1.28 \pm 0.14$ \\
\hline
\end{tabular}

NOTE. - ${ }^{\text {a }}$ Quadrature sum of INNER + 2 MIDDLE + 2 OUTER $M_{*}$ and SFR errors in parentheses; $M_{*}$ are consistent with lognormal estimates. ${ }^{\mathrm{b}}$ Magnification-corrected (Table 1); uncertainties incorporate $\mu$ errors. ${ }^{\mathrm{c}}$ Formal $2 \sigma$ limit.

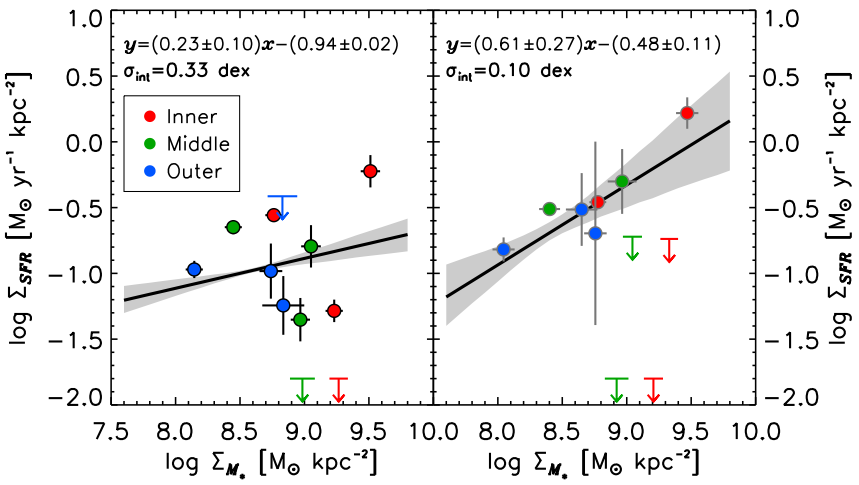

FIG. 20.- Resolved SFR vs. $M_{*}$ surface densities (analogous to Schmidt 1959; Kennicutt 1998) assuming lognormal (left) and delayed exponential SFHs (right). Both have $x \equiv \log \Sigma_{M_{*}}-8.75$, with linear best-fits performed only to detections by bootstrapping and varying points by their $x$ and $y$ errorbars. While quite different from each other, both inferences are similar to $z=0 \mathrm{IFU} /$ resolved SED estimates over a similar mass (but not $\Sigma_{M_{*}}$ ) range: Cano-Díaz et al. (2016) find a slope/dispersion of $0.68 \pm 0.04$ and $0.23 \mathrm{dex}$, respectively, corresponding to our delayed exponential results; Abdurro'uf \& Akiyama (2017) find 0.33 and 0.7 , respectively, closer to our lognormal results. This highlights the dependence of scaling relations on SFH choice.
NER levels are elevated and larger- $r$ values depressed relative to SSF's. The former effect is largely if not entirely due to CSF's AGN activity (Figures 3, 7), and higher resolution IFU spectroscopy will reveal if the latter is due to increased extinction towards its $\mathrm{H}$ II regions or a real suppression of sSFR beyond the factor of $\sim 3$ inferred from the full SED.

Finally, Figure 20 plots the full sample's 12 resolved $\Sigma_{\mathrm{SFR}}$ and $\Sigma_{M_{*}}$ values, a stellar mass reinterpretation of the SchmidtKennicutt Law (Schmidt 1959; Kennicutt 1998). The left panel shows lognormal inferences, with delayed exponentials at right. While these techniques yield quite different bestfit relations (Equations E1 and E2 below) both are nevertheless similar to previous $z=0$ IFU-/resolved SED based estimates: Cano-Díaz et al. (2016) find a slope and dispersion of $0.68 \pm 0.04$ and 0.23 dex, respectively, corresponding to our delayed exponential results, while Abdurro'uf \& Akiyama (2017), find 0.33 and 0.7 , respectively, closer to (though with larger scatter than) our lognormal results, highlighting the dependence of such scaling relations on SFH choice.

Using lognormal SFHs, we find:

$$
\begin{aligned}
\log \left[\frac{\Sigma_{\mathrm{SFR}}}{\mathrm{M}_{\odot} \mathrm{yr}^{-1} \mathrm{kpc}^{-2}}\right]= & -(0.94 \pm 0.02) \\
& +(0.2 \pm 0.1) \log \left[\frac{\Sigma_{M_{*}}}{10^{8.75} \mathrm{M}_{\odot} \mathrm{kpc}^{-2}}\right],
\end{aligned}
$$

with intrinsic scatter $\sigma_{\text {int }}=0.33$ dex.

Using delayed exponentials yields:

$$
\begin{aligned}
\log \left[\frac{\Sigma_{\mathrm{SFR}}}{\mathrm{M}_{\odot} \mathrm{yr}^{-1} \mathrm{kpc}^{-2}}\right]= & -(0.48 \pm 0.11) \\
& +(0.6 \pm 0.3) \log \left[\frac{\Sigma_{M_{*}}}{10^{8.75} \mathrm{M}_{\odot} \mathrm{kpc}^{-2}}\right]
\end{aligned}
$$

with intrinsic scatter $\sigma_{\text {int }}=0.10 \mathrm{dex}$

\section{REFERENCES}

Abdurro'uf, \& Akiyama, M. 2017, ArXiv e-prints, arXiv:1704.04571 Abramson, L. E., Gladders, M. D., Dressler, A., et al. 2015, ApJ, 801, L12 - 2016, ApJ, 832, 7

Abramson, L. E., Kelson, D. D., Dressler, A., et al. 2014, ApJ, 785, L36

Abramson, L. E., \& Morishita, T. 2016, ArXiv e-prints, arXiv:1608.07577

Aird, J., Coil, A. L., Moustakas, J., et al. 2012, ApJ, 746, 90

Allen, J. T., Croom, S. M., Konstantopoulos, I. S., et al. 2015, MNRAS, 446 1567

Barro, G., Faber, S. M., Pérez-González, P. G., et al. 2013, ApJ, 765, 104

Barro, G., Kriek, M., Pérez-González, P. G., et al. 2016, ArXiv e-prints, arXiv:1607.01011

Belli, S., Newman, A. B., \& Ellis, R. S. 2017, ApJ, 834, 18

Benson, A. J. 2012, New Astronomy, 17, 175

Bertin, E., \& Arnouts, S. 1996, A\&AS, 117, 393

Bezanson, R., van Dokkum, P. G., Tal, T., et al. 2009, ApJ, 697, 1290

Blanc, G. A., Heiderman, A., Gebhardt, K., Evans, II, N. J., \& Adams, J. 2009, ApJ, 704, 842

Brammer, G. B., van Dokkum, P. G., \& Coppi, P. 2008, ApJ, 686, 1503

Brammer, G. B., van Dokkum, P. G., Franx, M., et al. 2012, ApJS, 200, 13

Bruzual, G., \& Charlot, S. 2003, MNRAS, 344, 1000

Bundy, K., Bershady, M. A., Law, D. R., et al. 2015, ApJ, 798, 7

Calvi, R., Poggianti, B. M., \& Vulcani, B. 2011, MNRAS, 416, 727

Calzetti, D., Armus, L., Bohlin, R. C., et al. 2000, ApJ, 533, 682

Caminha, G. B., Grillo, C., Rosati, P., et al. 2017, A\&A, 600, A90

Cano-Díaz, M., Sánchez, S. F., Zibetti, S., et al. 2016, ApJ, 821, L26

Cappellari, M., Emsellem, E., Krajnović, D., et al. 2011, MNRAS, 413, 813

Castellano, M., Amorín, R., Merlin, E., et al. 2016, A\&A, 590, A31

Ciesla, L., Elbaz, D., \& Fensch, J. 2017, ArXiv e-prints, arXiv:1706.08531

Couch, W. J., \& Sharples, R. M. 1987, MNRAS, 229, 423

Croton, D. J., Springel, V., White, S. D. M., et al. 2006, MNRAS, 365, 11

Davé, R., Oppenheimer, B. D., \& Finlator, K. 2011, MNRAS, 415, 11

Diemer, B., Sparre, M., Abramson, L. E., \& Torrey, P. 2017, ApJ, 839, 26
Domínguez Sánchez, H., Pérez-González, P. G., Esquej, P., et al. 2016, MNRAS, 457, 3743

Dressler, A. 1980, ApJ, 236, 351

Dressler, A., \& Abramson, L. 2015, in IAU Symposium, Vol. 311, IAU

Symposium, ed. M. Cappellari \& S. Courteau, 140-145

Dressler, A., \& Gunn, J. E. 1983, ApJ, 270, 7

Dressler, A., Oemler, Jr., A., Poggianti, B. M., et al. 2004, ApJ, 617, 867

Dressler, A., Kelson, D. D., Abramson, L. E., et al. 2016, ApJ, 833, 251

Dutton, A. A., Conroy, C., van den Bosch, F. C., Prada, F., \& More, S. 2010

MNRAS, 407, 2

Eggen, O. J., Lynden-Bell, D., \& Sandage, A. R. 1962, ApJ, 136, 748

Feroz, F., \& Hobson, M. P. 2008, MNRAS, 384, 449

Förster Schreiber, N. M., Genzel, R., Bouché, N., et al. 2009, ApJ, 706, 1364

Fritz, J., Poggianti, B. M., Bettoni, D., et al. 2007, A\&A, 470, 137

Fritz, J., Poggianti, B. M., Cava, A., et al. 2014, A\&A, 566, A32

Gadotti, D. A. 2009, MNRAS, 393, 1531

Gallagher, III, J. S., Hunter, D. A., \& Tutukov, A. V. 1984, ApJ, 284, 544

Gallazzi, A., Bell, E. F., Zibetti, S., Brinchmann, J., \& Kelson, D. D. 2014 ApJ, 788, 72

Gallazzi, A., Charlot, S., Brinchmann, J., White, S. D. M., \& Tremonti,

C. A. 2005, MNRAS, 362, 41

Genel, S., Nelson, D., Pillepich, A., et al. 2017, ArXiv e-prints, arXiv: 1707.05327

Gladders, M. D., Oemler, A., Dressler, A., et al. 2013, ApJ, 770, 64

Goddard, D., Thomas, D., Maraston, C., et al. 2017, MNRAS, 466, 4731

Grillo, C., Suyu, S. H., Rosati, P., et al. 2015, ApJ, 800, 38

Grogin, N. A., Kocevski, D. D., Faber, S. M., et al. 2011, ApJS, 197, 35

Guglielmo, V., Poggianti, B. M., Moretti, A., et al. 2015, MNRAS, 450, 2749

Harrison, C. M. 2014, in IAU Symposium, Vol. 304, Multiwavelength AGN

Surveys and Studies, ed. A. M. Mickaelian \& D. B. Sanders, 284-290

Hoag, A., Huang, K.-H., Treu, T., et al. 2016, ApJ, 831, 182 
Hopkins, P. F., Kereš, D., Oñorbe, J., et al. 2014, MNRAS, 445, 581 Horne, K. 1986, PASP, 98, 609

Illingworth, G., Magee, D., Bouwens, R., et al. 2016, ArXiv e-prints, arXiv: 1606.00841

Ishigaki, M., Kawamata, R., Ouchi, M., et al. 2015, ApJ, 799, 12

Iyer, K., \& Gawiser, E. 2017, ApJ, 838, 127

Jauzac, M., Clément, B., Limousin, M., et al. 2014, MNRAS, 443, 1549

Johnson, T. L., Sharon, K., Bayliss, M. B., et al. 2014, ApJ, 797, 48

Johnson, T. L., Rigby, J. R., Sharon, K., et al. 2017a, ApJ, 843, L21

Johnson, T. L., Sharon, K., Gladders, M. D., et al. 2017b, ApJ, 843, 78

Jones, T., Wang, X., Schmidt, K. B., et al. 2015, AJ, 149, 107

Juneau, S., Bournaud, F., Charlot, S., et al. 2014, ApJ, 788, 88

Kauffmann, G., Heckman, T. M., White, S. D. M., et al. 2003, MNRAS, 341, 33

Kelson, D. D. 2014, ArXiv e-prints, arXiv:1406.5191

Kelson, D. D., Benson, A. J., \& Abramson, L. E. 2016, ArXiv e-prints, arXiv: 1610.06566

Kelson, D. D., Illingworth, G. D., Franx, M., \& van Dokkum, P. G. 2001, ApJ, 552, L17

-. 2006, ApJ, 653, 159

Kelson, D. D., Williams, R. J., Dressler, A., et al. 2014, ApJ, 783, 110

Kennicutt, Jr., R. C. 1998, ApJ, 498, 541

Kepner, J. V. 1999, ApJ, 520, 59

Kriek, M., van der Wel, A., van Dokkum, P. G., Franx, M., \& Illingworth, G. D. 2008, ApJ, 682, 896

Kriek, M., Shapley, A. E., Reddy, N. A., et al. 2015, ApJS, 218, 15

Lang, P., Wuyts, S., Somerville, R., et al. 2014, ArXiv e-prints, arXiv: 1402.0866

Larson, R. B., Tinsley, B. M., \& Caldwell, C. N. 1980, ApJ, 237, 692

Lee, S.-K., Ferguson, H. C., Somerville, R. S., Wiklind, T., \& Giavalisco, M. 2010, ApJ, 725, 1644

Lee-Brown, D. B., Rudnick, G. H., Momcheva, I. G., et al. 2017, ApJ, 844, 43

Lilly, S. J., \& Carollo, C. M. 2016, ApJ, 833, 1

Lotz, J. M., Koekemoer, A., Coe, D., et al. 2017, ApJ, 837, 97

Ma, C.-P., Greene, J. E., McConnell, N., et al. 2014, ApJ, 795, 158

Madau, P., \& Dickinson, M. 2014, ARA\&A, 52, 415

Magdis, G. E., Bureau, M., Stott, J. P., et al. 2016, MNRAS, 456, 4533

Markwardt, C. B. 2009, in Astronomical Society of the Pacific Conference Series, Vol. 411, Astronomical Data Analysis Software and Systems XVIII, ed. D. A. Bohlender, D. Durand, \& P. Dowler, 251

Mason, C. A., Treu, T., Fontana, A., et al. 2017, ApJ, 838, 14

McAlpine, S., Helly, J. C., Schaller, M., et al. 2016, Astronomy and Computing, 15, 72

McDermid, R. M., Alatalo, K., Blitz, L., et al. 2015, MNRAS, 448, 3484

Momcheva, I. G., Brammer, G. B., van Dokkum, P. G., et al. 2016, ApJS, 225, 27

Morishita, T., \& Ichikawa, T. 2016, ApJ, 816, 87

Morishita, T., Ichikawa, T., Noguchi, M., et al. 2015, ApJ, 805, 34

Morishita, T., Abramson, L. E., Treu, T., et al. 2017, ApJ, 835, 254

Muñoz, J. A., \& Peeples, M. S. 2015, MNRAS, 448, 1430

Nelson, E. J., van Dokkum, P. G., Momcheva, I. G., et al. 2016a, ApJ, 817, L9

Nelson, E. J., van Dokkum, P. G., Förster Schreiber, N. M., et al. 2016b, ApJ, 828, 27

Newman, A. B., Belli, S. \& Ellis, R. S. 2015, ApJ, 813, L7

Newman, A. B., Ellis, R. S., Andreon, S., et al. 2014, ApJ, 788, 51

Newman, A. B., Ellis, R. S., Bundy, K., \& Treu, T. 2012, ApJ, 746, 162

Nipoti, C., Treu, T., Leauthaud, A., et al. 2012, MNRAS, 422, 1714

Noeske, K. G., Weiner, B. J., Faber, S. M., et al. 2007, ApJ, 660, L43

Oemler, Jr, A., Abramson, L. E., Gladders, M. D., et al. 2016, ArXiv e-prints, arXiv: 1611.05932

Oemler, Jr., A., Dressler, A., Gladders, M. G., et al. 2013a, ApJ, 770, 63

-. 2013b, ApJ, 770, 61

Pacifici, C., Kassin, S. A., Weiner, B., Charlot, S., \& Gardner, J. P. 2013, ApJ, 762, L15

Pacifici, C., Kassin, S. A., Weiner, B. J., et al. 2016, ApJ, 832, 79

Peng, C. Y., Ho, L. C., Impey, C. D., \& Rix, H.-W. 2002, AJ, 124, 266
Peng, Y., Maiolino, R., \& Cochrane, R. 2015, Nature, 521, 192

Peng, Y.-j., Lilly, S. J., Kovač, K., et al. 2010, ApJ, 721, 193

Poggianti, B. M., Smail, I., Dressler, A., et al. 1999, ApJ, 518, 576

Poggianti, B. M., Calvi, R., Bindoni, D., et al. 2013, ApJ, 762, 77

Poggianti, B. M., Moretti, A., Gullieuszik, M., et al. 2017, ApJ, 844, 48

Postman, M., Coe, D., Benítez, N., et al. 2012, ApJS, 199, 25

Richard, J., Jauzac, M., Limousin, M., et al. 2014, MNRAS, 444, 268

Rigby, J. R., Johnson, T. L., Sharon, K., et al. 2017, ApJ, 843, 79

Salim, S., Rich, R. M., Charlot, S., et al. 2007, ApJS, 173, 267

Salpeter, E. E. 1955, ApJ, 121, 161

Sánchez, S. F., Kennicutt, R. C., Gil de Paz, A., et al. 2012, A\&A, 538, A8

Sanders, R. L., Shapley, A. E., Zhang, K., \& Yan, R. 2017, ArXiv e-prints, arXiv: 1708.04625

Schlafly, E. F., \& Finkbeiner, D. P. 2011, ApJ, 737, 103

Schmidt, K. B., Treu, T., Brammer, G. B., et al. 2014, ApJ, 782, L36

Schmidt, M. 1959, ApJ, 129, 243

Schreiber, C., Elbaz, D., Pannella, M., et al. 2016, ArXiv e-prints, arXiv:1601.04226

Shapley, A. E., Reddy, N. A., Kriek, M., et al. 2015, ApJ, 801, 88

Silk, J., \& Norman, C. 1981, ApJ, 247, 59

Speagle, J. S., Steinhardt, C. L., Capak, P. L., \& Silverman, J. D. 2014

ApJS, 214, 15

Spitler, L. R., Labbé, I., Glazebrook, K., et al. 2012, ApJ, 748, L21

Steidel, C. C., Rudie, G. C., Strom, A. L., et al. 2014, ApJ, 795, 165

Tacchella, S., Dekel, A., Carollo, C. M., et al. 2016, MNRAS, 457, 2790

Tacchella, S., Carollo, C. M., Renzini, A., et al. 2015, Science, 348, 314

Thomas, D., Maraston, C., Bender, R., \& Mendes de Oliveira, C. 2005, ApJ, 621,673

Tinsley, B. M. 1968, ApJ, 151, 547

Toft, S., Zabl, J., Richard, J., et al. 2017, Nature, 546, 510

Trager, S. C., Faber, S. M., Worthey, G., \& González, J. J. 2000, AJ, 120, 165

Trager, S. C., Worthey, G., Faber, S. M., Burstein, D., \& Gonzalez, J. J. 1998, ApJS, 116, 1

Treu, T., Ellis, R. S., Liao, T. X., et al. 2005, ApJ, 633, 174

Treu, T., Schmidt, K. B., Brammer, G. B., et al. 2015, ApJ, 812, 114

van de Sande, J., Kriek, M., Franx, M., et al. 2013, ApJ, 771, 85

van der Wel, A., Franx, M., van Dokkum, P. G., et al. 2014, ApJ, 788, 28

van der Wel, A., Noeske, K., Bezanson, R., et al. 2016, ApJS, 223, 29

van Dokkum, P. G., Leja, J., Nelson, E. J., et al. 2013, ApJ, 771, L35

van Dokkum, P. G. Nelson, E. J., Franx, M., et al. 2015, ApJ, 813, 23

Vogelsberger, M., Genel, S., Springel, V., et al. 2014, MNRAS, 444, 1518

Voit, G. M., Bryan, G. L., O'Shea, B. W., \& Donahue, M. 2015, ApJ, 808, L30

Vulcani, B., Poggianti, B. M., Fritz, J., et al. 2015a, ApJ, 798, 52

Vulcani, B., Treu, T., Schmidt, K. B., et al. 2015b, ApJ, 814, 161

-. 2016, ApJ, 833, 178

Vulcani, B., Treu, T., Nipoti, C., et al. 2017, ApJ, 837, 126

Wang, X., Hoag, A., Huang, K.-H., et al. 2015, ApJ, 811, 29

Wang, X., Jones, T. A., Treu, T., et al. 2017, ApJ, 837, 89

Weisz, D. R., Dolphin, A. E., Skillman, E. D., et al. 2014, ApJ, 789, 148

Whitaker, K. E., van Dokkum, P. G., Brammer, G., \& Franx, M. 2012, ApJ, 754, L29

Whitaker, K. E., van Dokkum, P. G., Brammer, G., et al. 2013, ApJ, 770, L39

Whitaker, K. E., Franx, M., Leja, J., et al. 2014, ApJ, 795, 104

Williams, R. J., Quadri, R. F., Franx, M., van Dokkum, P., \& Labbé, I. 2009. ApJ, 691, 1879

Worthey, G., Faber, S. M., \& Gonzalez, J. J. 1992, ApJ, 398, 69

Wuyts, E., Rigby, J. R., Gladders, M. D., et al. 2012a, ApJ, 745, 86

Wuyts, E., Rigby, J. R., Gladders, M. D., \& Sharon, K. 2014, ApJ, 781, 61

Wuyts, E., Rigby, J. R., Sharon, K., \& Gladders, M. D. 2012b, ApJ, 755, 73

Wuyts, S., Förster Schreiber, N. M., Nelson, E. J., et al. 2013, ApJ, 779, 135

York, D. G., Adelman, J., Anderson, Jr., J. E., et al. 2000, AJ, 120, 1579

Zitrin, A., Broadhurst, T., Umetsu, K., et al. 2009, MNRAS, 396, 1985

Zitrin, A., Meneghetti, M., Umetsu, K., et al. 2013, ApJ, 762, L30

Zolotov, A., Dekel, A., Mandelker, N., et al. 2015, MNRAS, 450, 2327 